\title{
A clumpy-cloud photon-dominated regions model of the global far-infrared line emission of the Milky Way
}

\author{
M. Cubick ${ }^{1}$, J. Stutzki ${ }^{1}$, V. Ossenkopf ${ }^{1,2}$, C. Kramer $^{1}$, and M. Röllig ${ }^{1,3}$ \\ 1 I. Physikalisches Institut, Universität zu Köln, Zülpicher Str. 77, 50937 Köln, Germany \\ e-mail: cubick@ph1.uni-koeln.de \\ 2 SRON Netherlands Institute for Space Research, PO Box 800, 9700 AV Groningen, The Netherlands \\ 3 Argelander Institut für Radioastronomie, Universität Bonn, Auf dem Hügel 71, 53121 Bonn, Germany
}

Received 18 December 2007 / Accepted 7 July 2008

\section{ABSTRACT}

\begin{abstract}
Context. The fractal structure of the interstellar medium suggests that the interaction of UV radiation with the ISM as described in the context of photon-dominated regions (PDR) dominates most of the physical and chemical conditions, and hence the far-infrared and submm emission from the ISM in the Milky Way.

Aims. We investigate to what extent the Galactic FIR line emission of the important species $\mathrm{CO}, \mathrm{C}, \mathrm{C}^{+}$, and $\mathrm{O}$, as observed by the Cosmic Background Explorer (COBE) satellite can be modeled in the framework of a clumpy, UV-penetrated cloud scenario.

Methods. The far-infrared line emission of the Milky Way is modeled as the emission from an ensemble of clumps with a power law clump mass spectrum and mass-size relation with power-law indices consistent with the observed ISM structure. The individual clump line intensities are calculated using the KOSMA- $\tau$ PDR-model for spherical clumps. The model parameters for the cylindrically symmetric Galactic distribution of the mass density and volume filling factor are determined by the observed radial distributions. A constant FUV intensity, in which the clumps are embedded, is assumed.

Results. We show that this scenario can explain, without any further assumptions and within a factor of about 2, the absolute FIR-line intensities and their distribution with Galactic longitude as observed by COBE.
\end{abstract}

Key words. ISM: clouds - Galaxy: disk - infrared: ISM - infrared: galaxies - submillimeter - ISM: structure

\section{Introduction}

The importance of the interaction between the interstellar UVradiation and dense clouds in the ISM, determining the physical and chemical conditions in the surface regions of molecular clouds, which are modeled as so-called photon-dominated regions (PDRs), has been recognized since the first observations of the dominant cooling lines in the FIR, [C II] $158 \mu \mathrm{m}$, (Russell et al. 1980, 1981; Stutzki et al. 1988; Mizutani et al. 1994), [O I] $63 \mu \mathrm{m}$ (Melnick et al. 1979; Stacey et al. 1983) and the midand high- $J$ CO lines (Storey et al. 1981; Watson et al. 1985; Jaffe et al. 1987; Boreiko \& Betz 1991). Starting from the first PDRmodels, tailored to explain the FIR line emission from massive star forming regions such as Orion (Tielens \& Hollenbach 1985; Sternberg \& Dalgarno 1989) the inclusion of more details of the heating and cooling mechanisms as well as of the chemical network, nowadays allows the modeling over a wide range of physical parameters and has resulted in successful modeling of many detailed aspects of observed photon-dominated regions (review by Hollenbach \& Tielens 1999). PDRs thus have proven to be a very useful concept in understanding the mutual interaction between star formation and the structure of the ISM, both in individual regions in the Milky Way, but also in star forming regions in external galaxies (Kramer et al. 2005; Contursi et al. 2002; Malhotra et al. 2001; Unger et al. 2000; Nikola et al. 1998; Madden et al. 1997; Schilke et al. 1993). An overview of the different models is given by the recent comparison study of PDR codes (Röllig et al. 2007).

The importance of the PDR scenario is related to the fact that the ISM is fractal and thus most of the material is close to surfaces and hence affected by UV radiation, i.e. is located in PDRs. This was realized early on by comparing the spatial distribution of observed PDR tracers (Stutzki et al. 1988; Howe et al. 1991) with simple models of homogeneous or clumpy cloud structures. The observed relatively uniform line ratios of low$J{ }^{13} \mathrm{CO}$ and ${ }^{12} \mathrm{CO}$ in molecular clouds, inconsistent with simple, uniform cloud models, are shown to be naturally explained if the emission is assumed to originate in many, relatively small clumps (Störzer et al. 1996). The necessity of high densities in order to explain the observed large ${ }^{13} \mathrm{CO}$ brightnesses in the mid$J$ lines in the submm independently indicate a clumpy structure (Graf et al. 1990; Wolfire et al. 1989).

The fractal structure of the ISM is well represented by an ensemble of clumps with a power law clump mass distribution, and a power law clump mass-size relation (Stutzki et al. 1998). Power law mass spectra have been derived by decomposing the observed 3D-datacubes of emission of CO isotopologues into clumps by various methods (Stutzki \& Güsten 1990; Kramer et al. 1998; Williams \& Blitz 1993; Williams et al. 1994). The identified clumps typically show also a power law mass-size relation, although obtaining significant coverage over more then 1.5 to 2 orders of magnitude in length scale is difficult; combining low and high angular resolution observations of the Polaris Flare, Heithausen et al. (1998) demonstrated a power law mass-size relation over 3 orders of magnitude in length scale. Calculating the PDR emission from an ensemble of clumps with a given mass spectrum and mass-size relation thus offers a convenient way to model the submillimeter and FIR line emission of the ISM as an UV penetrated clump ensemble with the given fractal characteristics. 
The far-infrared absolute spectrophotometer (FIRAS) on the COBE satellite conducted a spectral line survey of the Milky Way in the far-infrared region at wavelengths longward of $100 \mu \mathrm{m}$ (Wright et al. 1991; Bennett et al. 1994). The spatial and spectral resolutions of respectively $7^{\circ}$ and $0.45 \mathrm{~cm}^{-1}$ allowed detection of the $\mathrm{CO}$ rotational transitions from $J=1-0$ to $8-7$, and the fine structure transitions of [C II] $158 \mu \mathrm{m},[\mathrm{N} \mathrm{II}] 122$ and $205 \mu \mathrm{m}$, [O I] $146 \mu \mathrm{m}$, and [C I] 370 and $609 \mu \mathrm{m}$, in addition to the dust FIR continuum. The wavelength coverage of COBE FIRAS did not allow to detect a few other important cooling lines of the ISM like the [OI] line at $63 \mu \mathrm{m}$ or the [O III] line at $88 \mu \mathrm{m}$. Fixsen et al. (1999) report the distribution of spectral line emission along the Galactic plane. They re-binned the data to a $5^{\circ}$-grid in Galactic longitude and assumed an latitudinal extension of the FIR line emission of $1^{\circ}$ according to the results of the DIRBE FIR continuum observations. In a first attempt to interpret the observed emission with PDR models, they fit the plane-parallel PDR model of Hollenbach (1991) to the observed line ratios in the Galactic center region to derive a density of $30 \mathrm{~cm}^{-3}$ and FUV field of 10 Habing units ${ }^{1}$. Heiles (1994) used the COBE Milky Way data to analyze the properties of the extended ionized gas traced by the [N II] lines. Misiriotis et al. (2006) combined COBE DIRBE and FIRAS continuum data at wavelengths between $1 \mu \mathrm{m}$ and $1 \mathrm{~mm}$ wavelength to constrain exponential axisymmetric models for the spatial distribution of the dust, the stars, and the gas in the Milky Way.

In this paper we investigate to what degree the global FIRline emission distribution of the Milky Way as observed by COBE can be explained in the framework of an UV-penetrated, clumpy cloud scenario of the ISM. We mention that the [C I] 1-0 and 2-1 and CO 4-3 and 7-6 emission from a massive star forming region in the Carina nebula can be explained by the emission of clumpy PDRs as recently shown by Kramer et al. (2008). The paper is organized as follows: in Sect. 2 we shortly summarize the concepts to quantify the clumpy, fractal cloud structure and introduce the clumpy cloud PDR model. Section 3 discusses the line emissivity of the clump ensemble, in particular the dependence on varying mass limits of the clump distribution. In Sect. 4 the model results are compared with the observational data from COBE. A short conclusion with outlook is given in Sect. 5.

\section{The galaxy model: an UV-illuminated clump ensemble}

Modeling the large-scale Galactic FIR line emission as observed by $\mathrm{COBE}$ is done in the following steps:

- generate a large parameter grid for the emission of individual spherical clumps with the KOSMA- $\tau$ PDR-model, where the individual clumps are characterized by their mass, density (resp. size), and the UV-intensity that they are embedded in;

- generate a clump mass and size distribution at each Galactocentric radius from the observed mass density and volume filling factor distribution in the Milky Way, assuming an universal power law index for the clump mass and size spectra and applying adequate high and low mass cutoffs;

- estimate the Galactic radial distribution of the effective mean UV-field in which the dense ISM clumps are embedded and the average clump density in the ensemble respectively the volume filling factor of the clump ensemble;

11 Habing unit corresponds to the integrated flux in the wavelength range from 91.2 to $111.0 \mathrm{~nm}$ of $1.6 \times 10^{-3} \mathrm{erg} \mathrm{s}^{-1} \mathrm{~cm}^{-2}$ (Habing 1968).
- by combining the above, calculate the volume emissivity of the ISM originating from the UV illuminated clump ensemble as a function of Galactocentric radius in the Milky Way; - calculate the line emission versus Galactic longitude by line-of-sight integration over this emissivity in the direction towards the Earth.

We describe each of these steps in detail in the following.

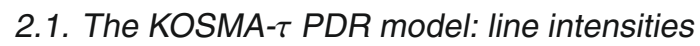 of an individual clump}

The KOSMA- $\tau$ PDR-model describes spherical clumps characterized by their mass, density (or size, respectively), and the incident UV field.

We treat the mass of the clumps $M_{\mathrm{cl}}$ as the total hydrogen gas mass. To obtain the total gas mass of a clump the mass of helium and heavier elements has to be added. This is a factor of about 1.4 higher (Anders \& Grevesse 1989). Due to the fact, that the massive clumps dominate the ensemble mass (see Sect. 2.2) and contain only a few percent of their gas mass in the atomic phase, we can neglect the contribution of the atomic gas so that the ensemble mass is basically given by the mass of molecular hydrogen.

The radial density structure $n(r)$ of the model clumps with radius $R_{\mathrm{cl}}$ is given by an inner core region with constant density for $r<0.2 R_{\mathrm{cl}}$ and a power law with index -1.5 for $0.2 R_{\mathrm{cl}}<r<$ $R_{\mathrm{cl}}$ :

$n(r)=n_{\mathrm{s}} \cdot \begin{cases}\left(\frac{r}{R_{\mathrm{cl}}}\right)^{-1.5}, & \text { for } 0.2 R_{\mathrm{cl}}<r<R_{\mathrm{cl}} \\ 5^{1.5}, & \text { for } r \leq 0.2 R_{\mathrm{cl}} .\end{cases}$

Note that $n_{\mathrm{s}}=n_{\mathrm{H}, \mathrm{s}}+2 n_{\mathrm{H}_{2}, \mathrm{~s}}$ is the total hydrogen nucleus number density at the clump surface. By neglecting the presence of helium and metals in the number density, we underestimate the number of collision partners by a factor of 1.2. The effect on the collision rates is, however, smaller due to the larger mass and thus lower velocity of helium. This does not change the results significantly. With the clump mass $M_{\mathrm{cl}}$, the clump volume $V_{\mathrm{cl}}=\frac{4 \pi}{3} R_{\mathrm{cl}}^{3}$, and the mass of the hydrogen atom $\mathrm{m}_{\mathrm{H}}$, the average clump density $n_{\mathrm{cl}}=M_{\mathrm{cl}} /\left(\mathrm{m}_{\mathrm{H}} V_{\mathrm{cl}}\right)$, used in the following to characterize each clump, is given by $n_{\mathrm{cl}}=\left(2-5^{-3 / 2}\right) n_{\mathrm{s}} \approx 1.91 n_{\mathrm{s}}$.

The FUV flux illuminating each clump is assumed to be isotropic. It is given in units of the Draine field $\chi_{\mathrm{D}}=2.7 \times 10^{-3} \mathrm{erg} \mathrm{s}^{-1} \mathrm{~cm}^{-2}$. This is the flux of the local interstellar radiation field (ISRF) integrated over the wavelength interval from 91.2 to $111.0 \mathrm{~nm}$ (Draine 1978).

In the KOSMA- $\tau$ PDR-model a constant flux of cosmic rays and the incident FUV radiation are the only heating sources of the clumps. The temperature and chemical structure of the clumps are derived from a radiative transfer computation including line-shielding for the incident FUV radiation and an escape probability approximation for the emitted FIR line radiation. The efficiency of the line cooling depends on the average line width $\delta v$, which is assumed to be $1.2 \mathrm{~km} \mathrm{~s}^{-1}$ (see Table 3). More details are described by Gierens et al. (1992), Köster et al. (1994), Störzer et al. (1996), and Röllig et al. (2006).

In order to calculate the average line intensity $I_{\mathrm{cl}}$ of each clump, first a line-of-sight integration of the source function and the attenuation along parallel paths through the clump is performed to calculate the resulting intensities $I(p)$ for different impact parameters $p$. As an example the line integrated CO 7-6 intensities are shown as a function of impact parameter in Fig. 1. Note the strong emission of the small, low mass, high density 


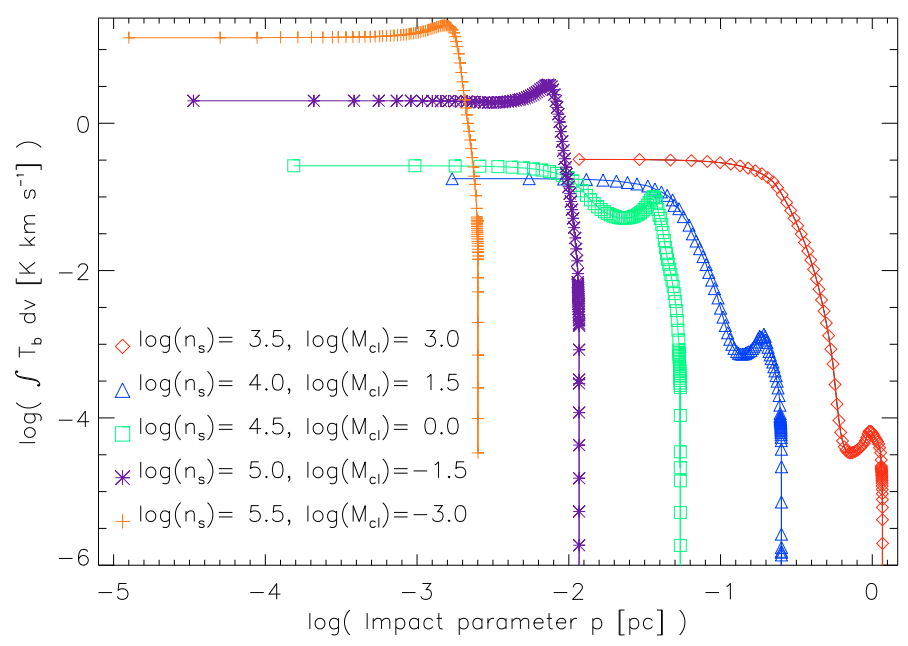

Fig. 1. Brightness temperature vs. impact parameter $p$ of the CO 7-6 rotational transition for selected clumps with mass and density values following the clump-mass spectrum and mass-size relation. $n_{\mathrm{s}}$ is the gas density at the surface of the clumps in $\left[\mathrm{cm}^{-3}\right]$ (see Eq. (1)), $M_{\mathrm{cl}}$ the clump mass in $\left[M_{\odot}\right]$, and the incident FUV-flux is $\chi=10^{2} \chi_{\mathrm{D}}$.

clumps. The physical reason for this is the enhanced population of the $J=7$ rotational excited state (i.e. a higher excitation temperature) of the $\mathrm{CO}$ molecule in the smaller and denser clumps, as only for those the density in the clump center falls near or above the critical density of the CO 7-6 rotational transition of about $10^{6} \mathrm{~cm}^{-3}$ (Kaufman et al. 1999; Kramer et al. 2004). The less dense but massive clumps have much larger column densities, partially compensating for the lower excitation temperature and increasing the optical depth. This leads to the apparently constant central brightness temperatures of the bigger clumps in Fig. 1. Detailed information about these clumps is compiled in Table 1.

Second, $I_{\mathrm{cl}}$ is obtained by averaging over the projected clump surface via

$I_{\mathrm{cl}}=\frac{2}{R_{\mathrm{cl}}^{2}} \int_{0}^{R_{\mathrm{cl}}} I(p) p \mathrm{~d} p$.

The results of these computations are used for the clump ensemble intensities.

We generate a large grid of intensities for the three independent clump parameters mass, density and FUV intensity, with grid points equidistant on a logarithmic scale in steps of half an order of magnitude. The range covered by the parameter cube of the KOSMA- $\tau$ PDR-model is shown in Table 2. For clumps with intermediate parameter values, the KOSMA- $\tau$ PDR-model intensities are interpolated between the grid points using logarithmic differences. Further parameters for the PDR model, held fixed for the present work, are listed in Table 3.

\subsection{Clump mass distribution and geometry}

Observations of the ISM show structure at all scales down to the resolution limit, as studied by e.g. Stutzki et al. (1988), Stutzki \& Güsten (1990), Heithausen et al. (1998), Kramer et al. (1998), Simon et al. (2001). Quantitative analysis of the observed intensity distribution shows that it can be described as a selfsimilar, i.e. fractal structure. Alternatively, the observed $3 \mathrm{~d}$ spectral line data cubes can be decomposed into clump ensembles.
The "clump-mass spectrum", i.e. the number of clumps $\mathrm{d} N_{\mathrm{cl}}$ per mass bin $\mathrm{d} M_{\mathrm{cl}}$, is then given by

$\frac{\mathrm{d} N_{\mathrm{cl}}}{\mathrm{d} M_{\mathrm{cl}}}=A M_{\mathrm{cl}}^{-\alpha}$

The size of the clumps is correlated with their mass via the "mass-size relation":

$M_{\mathrm{cl}}=C R_{\mathrm{cl}}^{\gamma}$.

The indices $\alpha$ and $\gamma$ of the clump ensemble are related to the fractional Brownian-motion power law index characterizing the fractal structure of the ISM (Stutzki et al. 1998). We choose values of 1.8 and 2.3, respectively, following the results from Heithausen et al. (1998). The ensemble mass $M_{\text {ens }}$ is obtained by integrating the clump-mass spectrum over a mass range from a low mass cutoff $m_{\mathrm{l}}$ to a high mass cutoff $m_{\mathrm{u}}$. The total mass of the clump ensemble $M_{\mathrm{ens}}=\int_{m_{\mathrm{l}}}^{m_{\mathrm{u}}} M_{\mathrm{cl}} \frac{\mathrm{d} N_{\mathrm{cl}}}{\mathrm{d} M_{\mathrm{cl}}} \mathrm{d} M_{\mathrm{cl}}$ determines the constant of proportionality $A$ in Eq. (3) via

$M_{\mathrm{ens}}=\frac{A}{2-\alpha}\left(m_{\mathrm{u}}^{2-\alpha}-m_{1}^{2-\alpha}\right)$.

Note that for $1<\alpha<2$ the bulk of the ensemble mass is contained in the high mass clumps. In contrast, the total number of clumps $N_{\mathrm{ens}}=\int_{m_{\mathrm{l}}}^{m_{\mathrm{u}}} \frac{\mathrm{d} N_{\mathrm{cl}}}{\mathrm{d} M_{\mathrm{cl}}} \mathrm{d} M_{\mathrm{cl}}=\frac{A}{1-\alpha}\left(m_{\mathrm{u}}^{1-\alpha}-m_{1}^{1-\alpha}\right)$ is dominated by the low mass clumps. Using the volume $V_{\mathrm{cl}}=\frac{4 \pi}{3} R_{\mathrm{cl}}^{3}$ of a clump of mass $M_{\mathrm{cl}}$ and the mass-size relation, the volume of the clump ensemble $V_{\mathrm{ens}}=\int_{m_{\mathrm{l}}}^{m_{\mathrm{u}}} \frac{\mathrm{d} N_{\mathrm{cl}}}{\mathrm{d} M_{\mathrm{cl}}} V_{\mathrm{cl}} \mathrm{d} M_{\mathrm{cl}}$ is given by

$V_{\mathrm{ens}}=\frac{A}{C^{3 / \gamma}} \frac{4 \pi}{3} \frac{m_{\mathrm{u}}^{1+3 / \gamma-\alpha}-m_{1}^{1+3 / \gamma-\alpha}}{1+3 / \gamma-\alpha}$

For the values of $\alpha$ and $\gamma$ given above, the exponent is $1+3 / \gamma-\alpha \approx 0.5$, and hence the ensemble volume is dominated by the large and most massive clumps.

The above applies as long as we are concerned with the integral properties of the clump distribution. In order to take the spatial distribution of the clumpy medium into account, we now switch to a volume specific notation where the medium is characterized by the (volume) number density, rather than the number, of the clumps, and by the mass density rather than the total mass. We obtain for the volume- and mass-differential clump distribution, i.e. for the (volume) number density of clumps $\mathrm{d} \tilde{N}_{\mathrm{cl}}$ per clump mass interval $\mathrm{d} M_{\mathrm{cl}}$, the "clump number density spectrum" as a function of Galactocentric radius $r$ :

$\frac{\mathrm{d} \tilde{N}_{\mathrm{cl}}(\boldsymbol{r})}{\mathrm{d} M_{\mathrm{cl}}}=\tilde{A}(\boldsymbol{r}) M_{\mathrm{cl}}^{-\alpha}$

The "clump mass density spectrum", i.e. the mass per volume element, per mass interval of the clump ensemble is correspondingly given by

$\frac{\mathrm{d} \tilde{N}_{\mathrm{cl}}(\boldsymbol{r})}{\mathrm{d} M_{\mathrm{cl}}} M_{\mathrm{cl}}=\tilde{A}(\boldsymbol{r}) M_{\mathrm{cl}}^{1-\alpha}$,

and the mass differential clump volume per volume element, i.e. the "clump filling factor spectrum", is

$\frac{\mathrm{d} \tilde{N}_{\mathrm{cl}}(\boldsymbol{r})}{\mathrm{d} M_{\mathrm{cl}}} V_{\mathrm{cl}}=\tilde{A}(\boldsymbol{r}) C^{-3 / \gamma}(\boldsymbol{r}) M_{\mathrm{cl}}^{3 / \gamma-\alpha}$ 
Table 1. Properties of the KOSMA- $\tau$ PDR-model clumps with given masses and densities at an incident FUV-flux of $10^{2} \chi_{\mathrm{D}}$. Orders of magnitude are given in parentheses.

\begin{tabular}{ccccccc}
\hline \hline \multicolumn{2}{c}{ Densities } & Mass & \multicolumn{3}{c}{ Column densities } & \multicolumn{2}{c}{ Temperatures } \\
Surface & Center & & \multicolumn{3}{c}{ Clump average } \\
$\log \left(n_{\mathrm{s}} / \mathrm{cm}^{-3}\right)$ & $\log \left(n_{\mathrm{c}} / \mathrm{cm}^{-3}\right)$ & $\log \left(M_{\mathrm{cl}} / M_{\odot}\right)$ & $\left\langle N_{\mathrm{H}_{2}}\right\rangle$ & $\left\langle N_{\mathrm{CO}}\right\rangle$ & $\left\langle T_{\mathrm{H}_{2}}\right\rangle$ & $\left\langle T_{\mathrm{CO}}\right\rangle$ \\
\hline 3.5 & 4.55 & 3.0 & $1.41(22)$ & $1.71(18)$ & 21.7 & 12.3 \\
4.0 & 5.05 & 1.5 & $9.74(21)$ & $1.50(18)$ & 23.8 & 11.0 \\
4.5 & 5.55 & 0.0 & $6.69(21)$ & $1.07(18)$ & 26.5 & 12.5 \\
5.0 & 6.05 & -1.5 & $4.57(21)$ & $7.21(17)$ & 29.6 & 15.5 \\
5.5 & 6.55 & -3.0 & $3.12(21)$ & $4.94(17)$ & 32.8 & 19.9 \\
\hline
\end{tabular}

Table 2. The parameter values of the precomputed KOSMA- $\tau$ PDRmodel clumps are equidistant on logarithmic scale in steps of half an order of magnitude.

\begin{tabular}{lc}
\hline \hline Quantity & Parameter range \\
\hline Density $n_{\mathrm{s}} / \mathrm{cm}^{-3}$ & $10^{2}, \ldots, 10^{6}$ \\
Mass $M_{\mathrm{cl}} / M_{\odot}$ & $10^{-3}, \ldots, 10^{3}$ \\
FUV flux $\chi / \chi_{\mathrm{D}}$ & $10^{0}, \ldots, 10^{6}$ \\
\hline
\end{tabular}

Integration over the clump mass leads to the "volume mass density" $\rho(\boldsymbol{r})$ and the "volume filling factor" $f_{V}(\boldsymbol{r})$, respectively, given by

$$
\begin{aligned}
\rho(\boldsymbol{r}) & =\frac{\tilde{A}(\boldsymbol{r})}{2-\alpha}\left(m_{\mathrm{u}}^{2-\alpha}-m_{1}^{2-\alpha}\right), \text { and } \\
f_{V}(\boldsymbol{r}) & =\frac{4 \pi}{3} \frac{\tilde{A}(\boldsymbol{r})}{C^{3 / \gamma}(\boldsymbol{r})} \frac{m_{\mathrm{u}}^{1+3 / \gamma-\alpha}-m_{1}^{1+3 / \gamma-\alpha}}{1+3 / \gamma-\alpha} .
\end{aligned}
$$

The "ensemble averaged clump mass density" $\rho_{\mathrm{ens}}(\boldsymbol{r})=$ $\rho(\boldsymbol{r}) / f_{V}(\boldsymbol{r})$ is given by

$\rho_{\mathrm{ens}}(\boldsymbol{r})=\frac{3}{4 \pi} C^{3 / \gamma}(\boldsymbol{r}) \frac{1+3 / \gamma-\alpha}{2-\alpha} \cdot \frac{\left(m_{\mathrm{u}}^{2-\alpha}-m_{1}^{2-\alpha}\right)}{\left(m_{\mathrm{u}}^{1+3 / \gamma-\alpha}-m_{1}^{1+3 / \gamma-\alpha}\right)}$.

As discussed in detail in Sect. 2.4 below, the distribution of the dense ISM in the Milky Way in our model is specified by the volume mass density $\rho(\boldsymbol{r})$ and the (higher) ensemble averaged clump mass density $\rho_{\text {ens }}(\boldsymbol{r})$ (respectively the volume filling factor of the molecular material). For given values of the low and high mass cutoffs of the clump distribution, Eqs. (10) and (12) then determine the pre-factor of the clump number density spectrum and its spatial variation,

$\tilde{A}(\boldsymbol{r})=\frac{2-\alpha}{m_{\mathrm{u}}^{2-\alpha}-m_{1}^{2-\alpha}} \rho(\boldsymbol{r})$,

through the volume mass density profile of the Milky Way, and the pre-factor of the mass-size relation and its spatial variation,

$C(\boldsymbol{r})=\left(\frac{4 \pi}{3} \frac{2-\alpha}{1+3 / \gamma-\alpha} \frac{m_{\mathrm{u}}^{1+3 / \gamma-\alpha}-m_{1}^{1+3 / \gamma-\alpha}}{m_{\mathrm{u}}^{2-\alpha}-m_{1}^{2-\alpha}} \rho_{\mathrm{ens}}(\boldsymbol{r})\right)^{\gamma / 3}$

through the ensemble averaged clump mass density.

A further quantity of interest is the beam filling factor $f_{\mathrm{B}}$, as the ratio of the solid angle filled by the clumps of the ensemble $\Omega_{\mathrm{ens}}$ and the solid angle of the beam $\Omega_{\mathrm{B}}$. The solid angle of a specific clump with mass $M_{\mathrm{cl}}$ and Radius $R_{\mathrm{cl}}$ at a distance $s=|s|$ (considering $s$ as given in Galactic coordinates centered at the sun) is given by

$\Omega_{\mathrm{cl}}(s)=2 \pi\left(1-\sqrt{1-\left(R_{\mathrm{cl}} / s\right)^{2}}\right) \simeq \pi \frac{R_{\mathrm{cl}}^{2}}{s^{2}}$,
Table 3. The fixed parameter values of the precomputed KOSMA- $\tau$ PDR-model clumps. Note that powers of ten are given in parentheses and the elemental abundances are given in units of the hydrogen abundance, i.e. $X_{i}=n_{i} / n$.

\begin{tabular}{lr}
\hline \hline Quantity & Value \\
\hline He abundance $X_{\mathrm{He}}$ & 0.1 \\
$\mathrm{O}$ abundance $X_{\mathrm{O}}$ & $3.0(-4)$ \\
$\mathrm{C}$ abundance $X_{\mathrm{C}}$ & $1.4(-4)$ \\
$\mathrm{S}$ abundance $X_{\mathrm{S}}$ & $2.8(-5)$ \\
${ }^{13} \mathrm{C}$ abundance $X_{{ }^{13} \mathrm{C}}$ & $2.1(-6)$ \\
Cosmic ray ionization rate $\chi_{\mathrm{CR}}\left[\mathrm{s}^{-1}\right]$ & $5(-17)$ \\
Velocity width $(F W H M) \delta v\left[\mathrm{~km} \mathrm{~s}^{-1}\right]$ & 1.2 \\
Dust UV cross section $\sigma_{\text {dust }}\left[\mathrm{cm}^{2}\right]$ & $1.9(-21)$ \\
\hline
\end{tabular}

for $R_{\mathrm{cl}} \ll s$. Then, the "solid angle density spectrum", i.e. the solid angle per volume and clump mass element of the ensemble, is

$\omega_{\mathrm{cl}}(\boldsymbol{s})=\frac{\mathrm{d} \tilde{N}_{\mathrm{cl}}}{\mathrm{d} M_{\mathrm{cl}}} \Omega_{\mathrm{cl}} \simeq \pi \frac{\tilde{A}(\boldsymbol{s})}{C^{2 / \gamma}(\boldsymbol{s}) s^{2}} M_{\mathrm{cl}}^{2 / \gamma-\alpha}$.

For the adopted values of $\alpha$ and $\gamma$ the exponent $2 / \gamma-\alpha$ has a value of -0.93 , implying that on a logarithmic mass scale, $\omega_{\mathrm{cl}}(s) \mathrm{d} M_{\mathrm{cl}} / \mathrm{d}\left(\ln M_{\mathrm{cl}}\right)=M_{\mathrm{cl}} \omega_{\mathrm{cl}}(s) \propto M_{\mathrm{cl}}^{0.07}$, each logarithmic mass interval contributes about equal to the solid angle of the ensemble. Integration over the given clump mass range gives the "solid angle density" of the ensemble

$$
\begin{aligned}
& \omega_{\mathrm{ens}}(\boldsymbol{s})=\int_{m_{\mathrm{l}}}^{m_{\mathrm{u}}} \omega_{\mathrm{cl}}(\boldsymbol{s}) \mathrm{d} M_{\mathrm{cl}} \\
& \simeq \pi \frac{\tilde{A}(s)}{C^{2 / \gamma}(s) s^{2}} \frac{m_{\mathrm{u}}^{2 / \gamma-\alpha+1}-m_{1}^{2 / \gamma-\alpha+1}}{2 / \gamma-\alpha+1} \\
& =\pi^{1 / 3}\left(\frac{3}{4}\right)^{2 / 3} \frac{\rho(s)}{\rho_{\mathrm{ens}}(s)^{2 / 3} s^{2}} \frac{(2-\alpha)^{1 / 3}(1+3 / \gamma-\alpha)^{2 / 3}}{2 / \gamma-\alpha+1} \\
& \cdot \frac{m_{\mathrm{u}}^{2 / \gamma-\alpha+1}-m_{1}^{2 / \gamma-\alpha+1}}{\left(m_{\mathrm{u}}^{2-\alpha}-m_{1}^{2-\alpha}\right)^{1 / 3}\left(m_{\mathrm{u}}^{1+3 / \gamma-\alpha}-m_{1}^{1+3 / \gamma-\alpha}\right)^{2 / 3}} \text {. }
\end{aligned}
$$

For the values of $\alpha$ and $\gamma$ adopted above, the nominator shows again a very weak mass dependence of $\propto m^{0.07}$. In the denominator in both factors the upper-mass cut-off dominates, so that the total dependence of the solid angle volume density of the clump ensemble is $\propto m_{\mathrm{u}}^{-(4 / 3-\alpha+2 / \gamma)} \simeq m_{\mathrm{u}}^{-0.4}$, i.e. weakly decreasing with increasing upper mass cut-off and basically independent on the lower mass cut-off. Integration over the beam volume $V_{\mathrm{B}}$ gives 
Table 4. Parameters of the Galaxy model (values at the solar circle).

\begin{tabular}{lllrl}
\hline \hline Name & Symbol & Unit & Value & Reference \\
\hline Radial extension of Galactic disk & $R_{\mathrm{G}}$ & $\mathrm{kpc}$ & 18 & {$[1]$} \\
Solar distance to Galactic center & $R_{\odot}$ & $\mathrm{kpc}$ & 8.5 & {$[1]$} \\
Half height $\mathrm{H}_{2} * *$ & $h_{\mathrm{H}}$ & $\mathrm{pc}$ & 59 & {$[1]$} \\
Half height CNM/WNM** & $h_{\mathrm{HI}}$ & $\mathrm{pc}$ & 115 & {$[1]$} \\
Half height WIM** & $h_{\mathrm{WIM}}$ & $\mathrm{pc}$ & $10^{3}$ & {$[2]$} \\
$\mathrm{H}_{2}$ mass surface density* & $\Sigma_{\mathrm{H}_{2}}$ & $M_{\odot} \mathrm{pc}^{-2}$ & 1.4 & {$[1]$} \\
CNM mass surface density* & $\Sigma_{\mathrm{CNM}}$ & $M_{\odot} \mathrm{pc}^{-2}$ & 2.25 & {$[1]$} \\
WNM mass surface density* & $\Sigma_{\mathrm{WNM}}$ & $M_{\odot} \mathrm{pc}^{-2}$ & 2.75 & {$[1]$} \\
WIM mass surface density* & $\Sigma_{\mathrm{WIM}}$ & $M_{\odot} \mathrm{pc}^{-2}$ & 1.85 & {$[2,1]$} \\
Ensemble averaged clump density* & $n_{\mathrm{ens}}$ & $\mathrm{cm}^{-3}$ & $10^{3.8}$ & \\
CNM density* & $n_{\mathrm{CNM}}$ & $\mathrm{cm}^{-3}$ & 32.9 & {$[1]$} \\
WNM/WIM density* & $n_{\mathrm{WNM}}$ & $\mathrm{cm}^{-3}$ & 0.349 & {$[1]$} \\
CNM temperature* & $T_{\mathrm{CNM}}$ & $\mathrm{K}$ & 85 & {$[1]$} \\
WNM/WIM temperature* & $T_{\mathrm{WNM}}$ & $\mathrm{K}$ & 7860 & {$[1]$} \\
FUV-flux** & $\chi$ & $\chi_{\mathrm{D}}$ & $10^{1.8}$ & \\
Metallicity** & $Z$ & $\mathrm{Z}_{\odot}$ & 1 & \\
Clump mass spectral index $* *$ & $\alpha$ & & 1.8 & {$[3]$} \\
Mass-size relation index** & $\gamma$ & & 2.3 & {$[3]$} \\
Upper clump mass limit** & $m_{\mathrm{u}}$ & $M_{\odot}$ & $10^{2}$ & {$[3]$} \\
Lower clump mass limit** & $m_{\mathrm{l}}$ & $M_{\odot}$ & $10^{-3}$ & {$[3]$} \\
\hline
\end{tabular}

* For distribution over Galactocentric radius see Fig. 2 or text.

** Assumed to be constant over the whole Galactic disk (see text). [1] Wolfire et al. (2003); [2] Reynolds (1991); [3] Heithausen et al. (1998).

the total solid angle filled by the clump ensemble

$$
\begin{aligned}
\Omega_{\mathrm{ens}}=\int_{V_{\mathrm{B}}} \omega_{\mathrm{ens}}(\boldsymbol{s}) \mathrm{d} V \\
\simeq \pi^{1 / 3}\left(\frac{3}{4}\right)^{2 / 3} \int_{V_{\mathrm{B}}} \frac{\rho(\boldsymbol{s})}{\rho_{\mathrm{ens}}(\boldsymbol{s})^{2 / 3} s^{2}} \mathrm{~d} V \\
\quad \cdot \frac{(2-\alpha)^{1 / 3}(1+3 / \gamma-\alpha)^{2 / 3}}{2 / \gamma-\alpha+1} \\
\quad \frac{m_{\mathrm{u}}^{2 / \gamma-\alpha+1}-m_{1}^{2 / \gamma-\alpha+1}}{\left(m_{\mathrm{u}}^{2-\alpha}-m_{1}^{2-\alpha}\right)^{1 / 3}\left(m_{\mathrm{u}}^{1+3 / \gamma-\alpha}-m_{1}^{1+3 / \gamma-\alpha}\right)^{2 / 3}},
\end{aligned}
$$

with the same dependence on upper and lower mass cut-off as for the volume specific solid angle.

\subsection{Volume emissivity and intensity of the clumpy medium}

In order to calculate the intensity of the clump ensemble, we neglect any absorption (in particular self-absorption in the spectral lines) among different clumps along the line-of-sight. This assumption may be violated locally in the high densities of individual star forming regions, although even there, the higher velocity dispersion of the virialized clump ensemble helps to avoid line-of-sight crowding in each velocity interval. It is reasonable for the large scale FIR line emission of the Galaxy, taking the additional velocity spread between individual regions due to the differential Galactic rotation into account. The intensity then simply is given by the line-of-sight integration over the volume emissivity of the clump ensemble

$I=\int_{1} \eta(s) \mathrm{d} s$

The beam average intensity then is

$I_{\mathrm{B}}=\frac{1}{\Omega_{\mathrm{B}}} \int_{\Omega_{\mathrm{B}}} \int_{1} \eta(s) \mathrm{d} s \mathrm{~d} \Omega=\frac{1}{\Omega_{\mathrm{B}}} \int_{V_{\mathrm{B}}} \eta(s) \frac{\mathrm{d} V}{s^{2}}$.

The KOSMA- $\tau$ PDR-model gives the clump average specific intensity $I_{\mathrm{cl}}$ for each clump of a given mass. The "volume emissivity spectrum" then is given by multiplication with the clump projected area $A_{\mathrm{cl}}=s^{2} \Omega_{\mathrm{cl}}$ and the clump number density spectrum, namely

$\frac{\mathrm{d} \eta(s)}{\mathrm{d} M_{\mathrm{cl}}}=\frac{\mathrm{d} \tilde{N}_{\mathrm{cl}}}{\mathrm{d} M_{\mathrm{cl}}} s^{2} \Omega_{\mathrm{cl}} I_{\mathrm{cl}}$.

With $\omega_{\mathrm{cl}}=\frac{\mathrm{d} \tilde{N}_{\mathrm{cl}}}{\mathrm{d} M_{\mathrm{cl}}} \Omega_{\mathrm{cl}}$, we obtain for the contribution to the ensemble intensity in the mass bin $\mathrm{d} M_{\mathrm{cl}}$

$\frac{\mathrm{d} I_{\mathrm{B}}}{\mathrm{d} M_{\mathrm{cl}}}=\frac{1}{\Omega_{\mathrm{B}}} \int_{V_{\mathrm{B}}} I_{\mathrm{cl}} \omega_{\mathrm{cl}}(\boldsymbol{s}) \mathrm{d} V$.

Integration over the mass ensemble results in the beam averaged intensity of the ensemble

$I_{\mathrm{B}}=\frac{1}{\Omega_{\mathrm{B}}} \int_{V_{\mathrm{B}}} \int_{m_{1}}^{m_{\mathrm{u}}} I_{\mathrm{cl}} \omega_{\mathrm{cl}}(\boldsymbol{s}) \mathrm{d} M_{\mathrm{cl}} \mathrm{d} V$.

Particularly, we obtain for a constant clump intensity the resulting beam average intensity $I_{\mathrm{B}}$ with the beam filling factor $f_{\mathrm{B}}=\Omega_{\mathrm{ens}} / \Omega_{\mathrm{B}}$ as $I_{\mathrm{B}}=f_{\mathrm{B}} I_{\mathrm{cl}}$.

\subsection{Galactic parameter distributions}

The Galactic parameter distributions of mass, density, and UVintensity are assumed to vary only in Galactocentric radial direction within a cylindrically symmetric Galactic disk of constant half-height $h$. The values of the different parameters (at the solar circle at $R=R_{\odot}=8.5 \mathrm{kpc}$ ) are compiled in Table 4 . We adopt a half-height of the molecular disk of $59 \mathrm{pc}$ and for the atomic gas layer of 115 pc (Wolfire et al. 2003). The fractal characteristics $\left(\alpha, \gamma, m_{1}, m_{\mathrm{u}}\right)$ of the clump distribution are assumed to be constant over the whole Galaxy. Due to the simplified structure of the Galactic parameter distributions in our model disk we do not expect to reproduce individual emission features along the Galactic plane. The Galactic mass distribution of the neutral ISM is a key parameter of the model, because the modeled line intensities are basically proportional to the mass of the molecular gas assumed to be completely represented by PDRs (see Sect. 1). The Galactic mass distribution has been discussed in the literature e.g. by Clemens (1985), Rohlfs \& Kreitschmann (1987), 
Williams \& McKee (1997), Bronfman et al. (2000). We use an approximation of the radial Galactic $\mathrm{H}_{2}$ mass distribution presented by Wolfire et al. (2003) for $3<R_{\mathrm{G}} / \mathrm{kpc}<18$. According to Williams \& McKee (1997, Fig. 3) the $\mathrm{H}_{2}$ mass surface density decreases inwards from 3 to $1.7 \mathrm{kpc}$ Galactocentric radius exponentially by a factor of about $3 / 8$. For $0.6<R_{\mathrm{G}} / \mathrm{kpc}<1.7$ the molecular hydrogen gas mass distribution is set constant. In the Galactic center region $\left(R_{\mathrm{G}}<0.6 \mathrm{kpc}\right)$, the molecular hydrogen gas mass surface density is assumed to have a constant value of $88.4 M_{\odot} \mathrm{pc}^{-2}$ corresponding to an $\mathrm{H}_{2}$ mass of $10^{8} M_{\odot}$ within the central $600 \mathrm{pc}$ of the Galaxy (Dahmen et al. 1998; Güsten \& Philipp 2004). This translates into a mean $\mathrm{H}_{2}$ gas mass density of $0.75 M_{\odot} \mathrm{pc}^{-3}$, corresponding to an $\mathrm{H}_{2}$ number density of about $15 \mathrm{~cm}^{-3}$. The assumed mass distributions are visualized in Fig. 2a). The neutral atomic hydrogen mass distribution is used to estimate the contribution of diffuse atomic phases to the [C II] $158 \mu \mathrm{m}$ fine structure line emission (as shown in Sect. 4.2.1). The contribution of the diffuse WIM is estimated by Reynolds (1990) to $37 \%$ of the total H I mass, which is slightly less than the WNM mass.

We adopt a lower clump mass limit $m_{1}$ of $10^{-3} M_{\odot}$ according to the observational lower clump mass limit from Heithausen et al. (1998), as well as an upper clump mass limit $m_{\mathrm{u}}$ of $10^{2} M_{\odot}$. Gorti \& Hollenbach (2002) show an decreasing lifetime of clumps with decreasing mass and increasing density and argue against the existence of stable, small, and dense PDR clumps. This indicates a transient nature of these clumps.

The observed mean gas density of molecular clouds shows variation with Galactocentric radius (e.g. Brand \& Wouterloot 1995). We adopt the Galactic radial distribution of the CNM density provided by Wolfire et al. (2003) for the ensemble averaged clump density $n_{\mathrm{ens}}=\rho_{\mathrm{ens}} / \mathrm{m}_{\mathrm{H}}$, scaled to an absolute value of $10^{3.8} \mathrm{~cm}^{-3}$ at the solar circle (Fig. 2b), obtained by a $\chi^{2}$ fit to the intensity distributions as observed by COBE (see Sect. 4.1). As shown in Sect. 2.2, the volume filling factor is determined by the underlying mass and density distributions. The result is shown in Fig. 2c). The computed beam filling factors from the solar point of view along the Galactic plane are shown in Fig. 2d). The density and temperature distributions with Galactocentric radius of the CNM and WNM, used in the following to estimate the [C II] emission from these phases, are adopted from Wolfire et al. (2003) (extrapolated inwards of $3 \mathrm{kpc}$ Galactocentric radius) and the WIM distributions are assumed to equal the WNM distributions (McKee \& Ostriker 1977; Reynolds 1991; Cox 2005; Hill et al. 2007).

The FUV flux in the Galaxy is mainly produced by OB stars. Observations show, that $\mathrm{OB}$ associations are associated with giant molecular clouds (GMCs) (e.g. Stark \& Blitz 1978). McKee $\&$ Williams (1997) and Williams \& McKee (1997) show, that the distributions of Galactic OB associations and GMCs can be described by truncated power laws with the same slope, i.e. show a strong correlation. Misiriotis et al. (2006) derive a nearly constant dust temperature from the COBE observations. Assuming a proportionality between dust continuum emission tracing the molecular material and the incident FUV flux from the stellar population this leads to a rather constant average FUV-flux impinging on the molecular gas within the Galactic disk. According to McKee \& Williams (1997, and references therein) there are 6-7 OB associations with more than 30 massive stars within $1 \mathrm{kpc}$ distance from the sun, dominating the local interstellar FUV radiation field. Blaauw (1985) states a mean scale height of about $50 \mathrm{pc}$ of the local OB associations. Therefore we assume one layer of surrounding OB associations with 3 next neighbors. This leads to an average radius of major influence for each $O B$
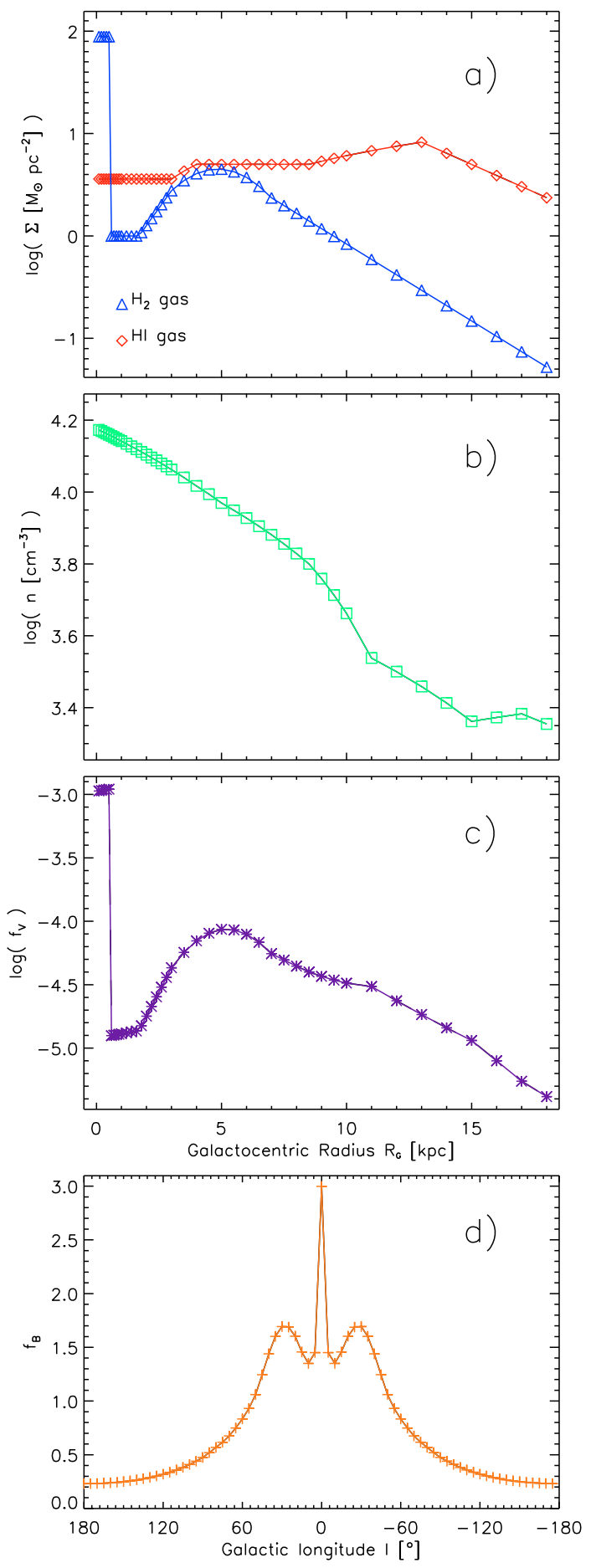

Fig. 2. a) Galactic mass surface density distributions of the molecular and atomic gas mass; b) ensemble averaged clump density distribution; c) resulting volume filling factor distribution with Galactocentric radius; d) derived beam filling factor distribution with Galactic longitude from the solar point of view.

association of $R_{\mathrm{OB}} \approx 7^{-1 / 2}$ to $6^{-1 / 2} \mathrm{kpc}$ or 378 to $408 \mathrm{pc}$. The actual distance measurement to the Orion Nebula Cluster resulting in $414 \pm 8$ pc (Menten et al. 2007) corresponds well to this range. Together with the local interstellar FUV-flux of $1 \chi_{\mathrm{D}}$ (Draine 1978) and average distances $d_{\mathrm{MC}-\mathrm{OB}}$ of about 20 to 50 pc of the GMCs to their associated OB associations (estimated from the sizes and shapes given in Stark \& Blitz 1978, Fig. 1 and Table 1) we obtain FUV-fluxes impinging the molecular clouds 

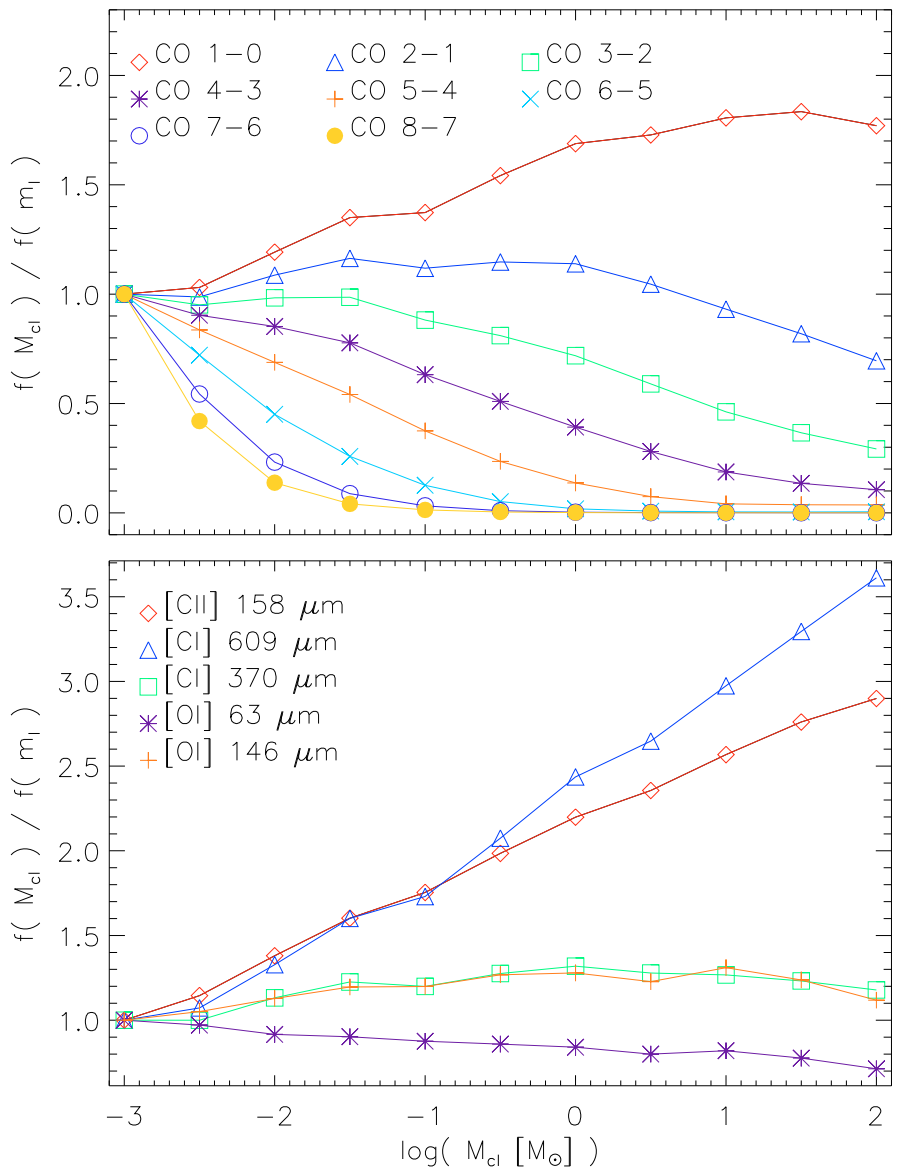

Fig. 3. Volume emissivity spectra of the clump ensemble for an ensemble averaged gas density of $n_{\text {ens }}=10^{3.8} \mathrm{~cm}^{-3}$ and an incident FUV-flux of $\chi=10^{1.8} \chi_{\mathrm{D}}$. According to the logarithmic scaling of the clump-mass axis $f\left(M_{\mathrm{cl}}\right)=M_{\mathrm{cl}} \mathrm{d} \eta / \mathrm{d} M_{\mathrm{cl}}$ is plotted to see the integral contribution of each mass interval. The values are normalized to the functional value at $M_{\mathrm{cl}}=m_{\mathrm{l}}$ for a convenient comparison of the different tracers.

of $\chi_{\mathrm{MC}} \approx\left(R_{\mathrm{OB}} / d_{\mathrm{MC}-\mathrm{OB}}\right)^{2} \chi_{\mathrm{D}} / 3$ with values of 19 to $139 \chi_{\mathrm{D}}$ or $\log \left(\chi_{\mathrm{MC}} / \chi_{\mathrm{D}}\right) \approx 1.3$ to 2.1 . in Sect. 4 we find a best fitting value of $\log \left(\chi / \chi_{\mathrm{D}}\right) \approx 1.8$ for our model, falling well within this range.

\section{The clump-ensemble emissivity}

To discuss and understand the behavior of the clump ensemble emissivity it is useful to first look at the features of individual clumps and the mass-differential emissivity of the ensemble. As explained in Sect. 2.3 the contribution to the volume emissivity spectrum of the ensemble $\mathrm{d} \eta / \mathrm{d} M_{\mathrm{cl}}$ in the various line transitions is dominated by the clump intensity $I_{\mathrm{cl}}$, as the filling factors do not significantly depend on the clump mass. The logarithmic volume emissivity spectrum $\mathrm{d} \eta / \mathrm{d} \log \left(M_{\mathrm{cl}}\right)=M_{\mathrm{cl}} \mathrm{d} \eta / \mathrm{d} M_{\mathrm{cl}}$ for an ensemble averaged clump density of $n_{\mathrm{ens}}=10^{3.8} \mathrm{~cm}^{-3}$ and an FUV-flux of $\chi=10^{1.8} \chi_{\mathrm{D}}$ is shown in Fig. 3. The symbols show the sampling points on the model grid. The upper plot shows the behavior for the different CO transitions. Note the trend towards larger contributions of the lower clump masses for higher transition numbers. The ensemble emission of the higher $\mathrm{CO}$ transitions $(J>5)$ almost exclusively stem from the low mass clumps. The bottom panel shows $M_{\mathrm{cl}} \mathrm{d} \eta / \mathrm{d} M_{\mathrm{cl}}$ for the fine structure cooling lines. The [C II] $158 \mu \mathrm{m}$ and [C I] $609 \mu \mathrm{m}$ emissions show an increased contribution of high mass clumps.

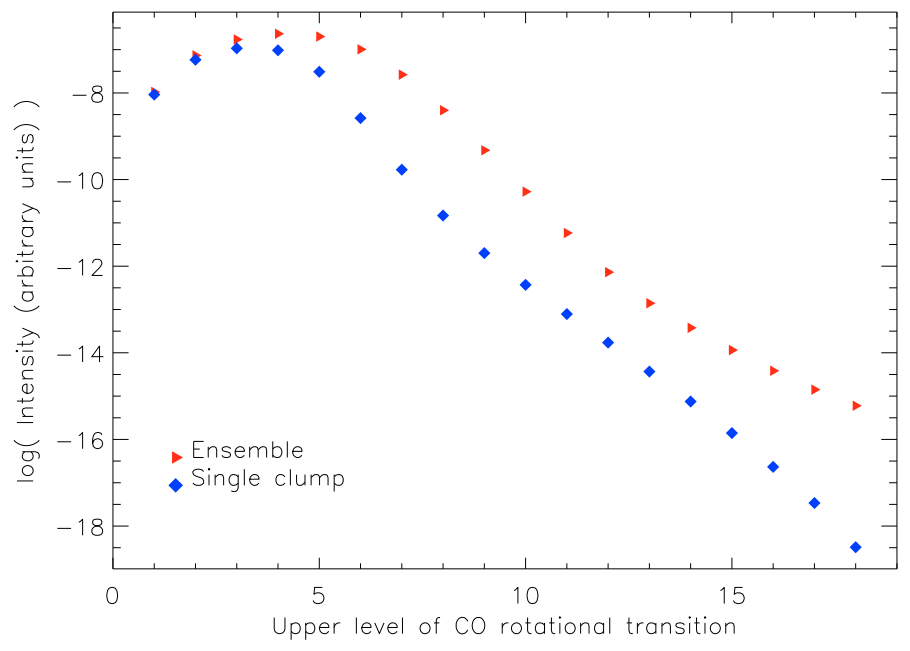

Fig. 4. The CO emission of a single clump of one solar mass and a density of $n_{\mathrm{cl}}=10^{3.8} \mathrm{~cm}^{-3}$ compared to the emission of an ensemble of clumps with the same total mass and ensemble averaged clump density, embedded in an isotropic FUV-flux of $10^{1.8} \chi_{\mathrm{D}}$.

In Fig. 4 a direct comparison of the emission of an individual clump with the emission of a clump ensemble with $1 M_{\odot}$ is shown for the different CO transitions. For $n_{\mathrm{ens}}=10^{3.8} \mathrm{~cm}^{-3}$ and $\chi=10^{1.8} \chi_{\mathrm{D}}$ we find a significantly higher intensity in the mid- $J$ CO transitions for the clump ensemble, which amounts to 2.5 orders of magnitude for the $\mathrm{CO} 8-7$ line. This is due to the fact, that the mid- $J \mathrm{CO}$ emission shows a steep decrease with clump mass (see Fig. 3).

Figure 5 illustrates the dependence of the normalized emissivity with variation of the lower and upper clump mass limits of the ensemble for different tracers. Again, the mid- $J$ CO emission shows a strong dependence on the lower clump mass limit, corresponding to the strong intensity enhancement with decreasing clump mass in these tracers. The $\mathrm{CO} 8-7$ emissivity is nearly proportional to the lower clump mass limit. The [C II] and [O I] emissivities show variations of one order of magnitude over an upper clump mass limit range of three orders of magnitude. This is due to the increasing surface to volume ratio of the clump ensemble with decreasing upper clump mass limit (cf. Sect. 2.2) and the fact that the [C II] and [OI] emissions arise mainly in shells in the outer parts of the clumps.

\section{Model results and comparison with observations}

The resulting emissivity distribution inherits the intensity dependence of the clumps on the Galactic mass and density distributions as shown in Fig. 6.

The beam averaged intensity is determined according to the derivation in Sect. 2.3. For the comparison of our model result with the COBE FIRAS observations we calculate the intensities in a beam with an extent of $5^{\circ}$ in Galactic longitude and $1^{\circ}$ in Galactic latitude (Fixsen et al. 1999). All main PDR cooling lines in the COBE FIRAS spectral range including [C II] $158 \mu \mathrm{m}$, [C I] 609 and $370 \mu \mathrm{m}, \mathrm{CO} J=1-0$ to $J=8-7$, and [O I] $146 \mu \mathrm{m}$ are used for the comparison.

To overview the emission features along the Galactic plane we labeled the main features visible in the [C II] intensity cut in Fig. 7. One can easily identify the Galactic large scale structure, dominated by the spiral arms and the Galactic molecular ring. 

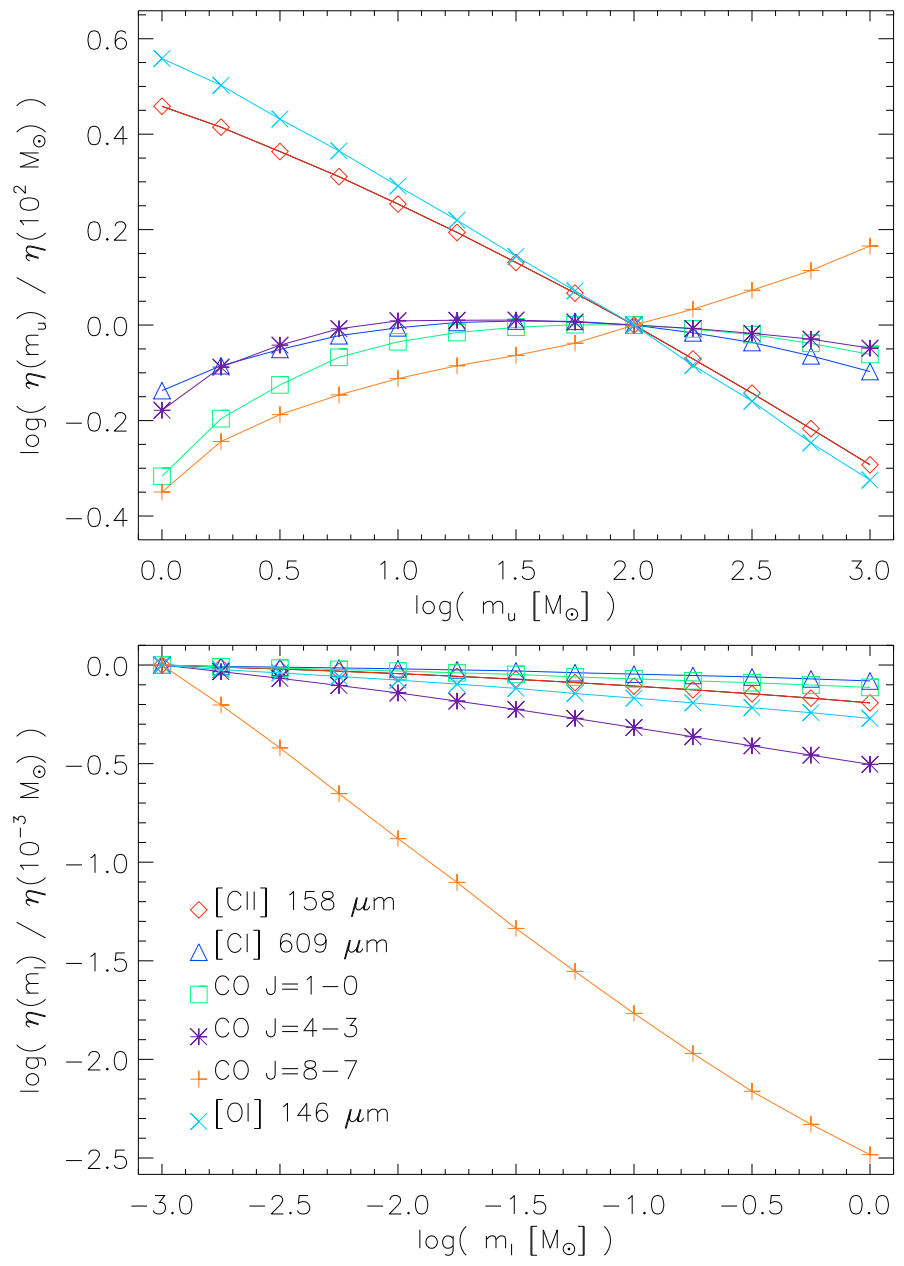

Fig. 5. Dependencies of the clump ensemble emissivity $\eta$ on the upper and lower clump mass, $m_{\mathrm{u}}$ respectively $m_{\mathrm{l}}$, for different tracers, $n_{\mathrm{ens}}=10^{3.8} \mathrm{~cm}^{-3}$, and $\chi=10^{1.8} \chi_{\mathrm{D}}$. The values are normalized to the upper $m_{\mathrm{u}}=10^{2} M_{\odot}$ respectively lower clump mass limit $m_{1}=10^{-3} M_{\odot}$, as used in the model.

\subsection{Quantitative comparison}

To quantify the quality of the model result we derive the reduced $\chi^{2}$ of the intensity distributions. The $\Delta I_{i}$ are the uncertainties of the observed intensities due to the calibration of the COBE FIRAS instrument and the data reduction. The number of degrees of freedom is given by $N_{\text {dof }}=N_{1} N_{\text {spec }}-2$, with $N_{1}$ the number of bins in Galactic longitude and $N_{\text {spec }}$ the number of species used for the fit. The subtraction of 2 is due to the 2 fitted parameters $n_{\text {ens }}$ and $\chi$. Hence, the reduced $\chi^{2}$ is given by

$\chi^{2}=\frac{1}{N_{\mathrm{dof}}} \sum_{i=1}^{N_{\mathrm{l}} N_{\mathrm{spec}}}\left(\frac{I_{\mathrm{obs}, i}-I_{\mathrm{mod}, i}}{\Delta I_{i}}\right)^{2}$.

The resulting $\chi^{2}$-distribution of the intensity distributions is plotted as contours onto the density-FUV-plane in Fig. 8. The minimum $\chi^{2}$ has a value of 16.5 at an ensemble averaged clump density at the solar circle of $n_{\mathrm{ens}}=10^{3.8} \mathrm{~cm}^{-3}$ and an FUV-flux of $\chi=10^{1.8} \chi_{\mathrm{D}}$. The $\chi^{2}$ distribution obviously confines the density to FUV-flux ratio $n_{\text {ens }} / \chi$ very well, as this ratio determines the molecular gas fraction within a PDR (Hollenbach \& Tielens 1999). In contrast, the product $n_{\mathrm{ens}} \chi$ is hardly constrained.

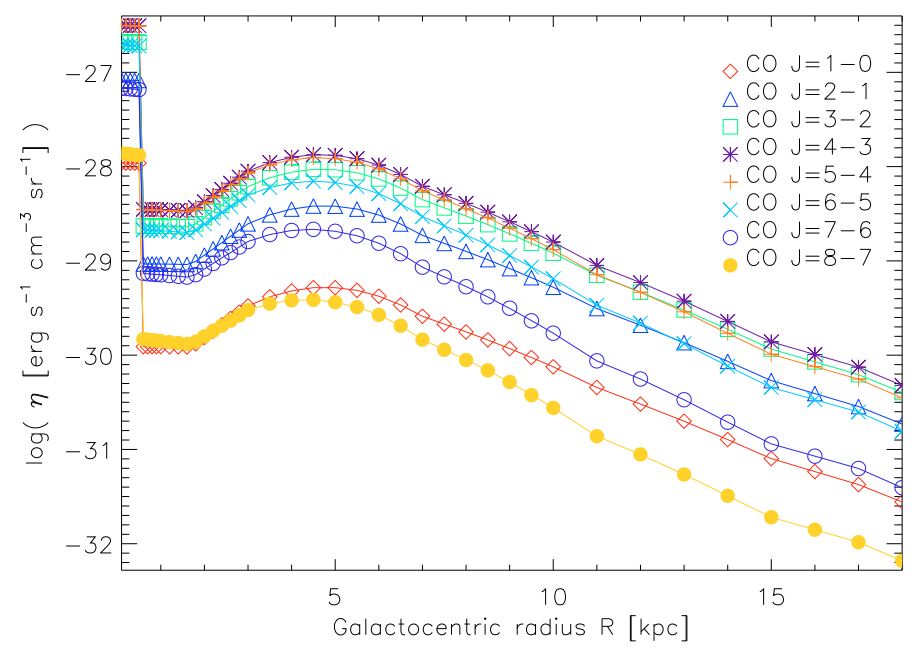

Fig. 6. Calculated $\mathrm{CO}$ emissivity distribution vs. Galactocentric radius for $n_{\text {ens }}=10^{3.8} \mathrm{~cm}^{-3}$ and $\chi=10^{1.8} \chi_{\mathrm{D}}$ at the solar circle.

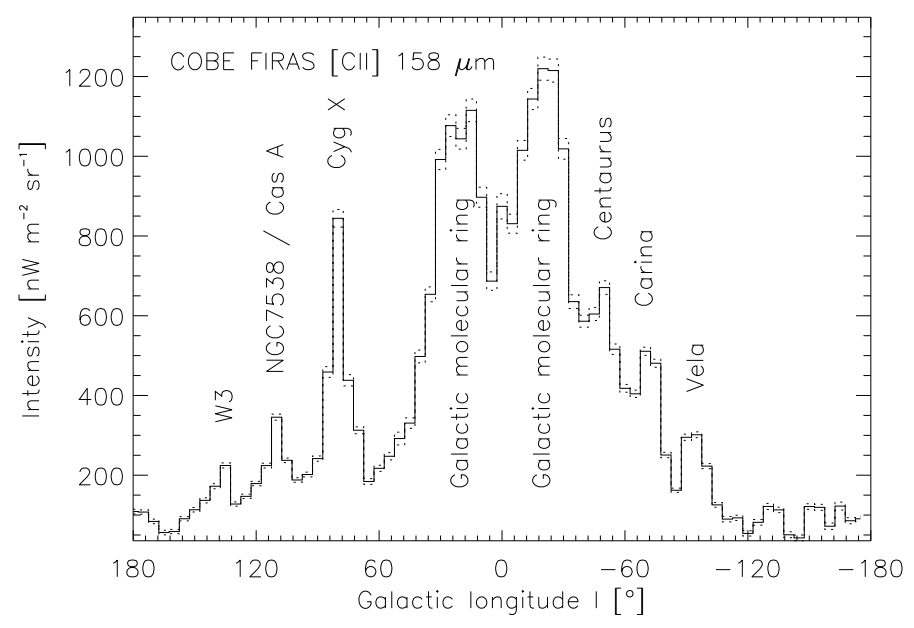

Fig. 7. The [C II] $158 \mu \mathrm{m}$ intensity distribution with Galactic longitude as published by Fixsen et al. (1999). The annotated source names are identified using the CO 1-0 map published by Dame et al. (2001).

\subsection{Discussion}

The results of the modeled line intensities are overlaid onto the observed intensities in Figs. 9 and 10. We note that the relatively high value of the minimum $\chi^{2}$ is due to the pronounced Galactic large scale structure in the observed [C II] emission (which is not contained in the smooth model distribution) and the high signal to noise ratio of these data. In fact, it is surprising that one can find average values for these parameters, which allow us to reproduce the bulk of the FIR line emission of the Milky Way. The partly significant local deviations of the model results from the observations are due to the strongly simplifying assumptions. Except CO $J=1-0,6-5$, and 8-7, which have large observational uncertainties, all lines are reproduced in total Galactic flux within factors below 2.3 ( $\mathrm{CO} J=2-1)$. The mismatch of the $\mathrm{CO} 2-1$ indicates an additional cold molecular cloud component in the Galactic gas distribution.

The resulting ${ }^{13} \mathrm{CO} 1-0 / \mathrm{CO} 1-0$ line intensity ratio with a value of about 4 is in accordance with observational results (Gierens et al. 1992; Falgarone et al. 1998). 


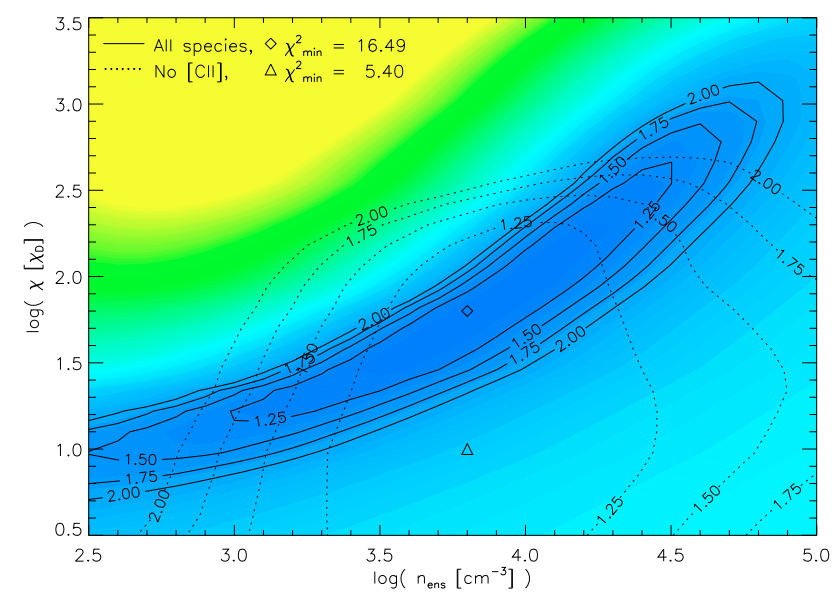

Fig. 8. The reduced $\chi^{2}$ plotted as contours in the ensemble averaged clump density-FUV-flux-plane. The contours are given as factors of the minimum $\chi^{2}$ value. The solid line as well as the color code represents the $\chi^{2}$ distribution including all species, while the dotted lines are the $\chi^{2}$ contours neglecting the $[\mathrm{C} \mathrm{II}]$ intensity. The $\chi_{\min }^{2}$ minima are given by the diamond and triangle symbols, respectively.

\subsubsection{Contributions to the Galactic [C II] $158 \mu \mathrm{m}$ emission}

We now turn to the comparison of the [C II] intensities as observed by COBE with the model results (see Fig. 9). Additional flux is measured mainly from the Galactic ring, the Cyg X region and other star-forming regions in the spiral arms. This is in contrast to the picture of [C II]-emission stemming to a large part from the CNM (Hollenbach \& Tielens 1999). Based on direct UV absorption measurements of the excited state of $\mathrm{C}^{+}$, Pottasch et al. (1979) and Gry et al. (1992) derive an average emissivity of the CNM, in particular diffuse $\mathrm{H}$ I-clouds, of $5 \times 10^{-26} \mathrm{erg} \mathrm{s}^{-1} \mathrm{H}$-atom ${ }^{-1}$. Using this number the contribution from PDRs to the Galactic [C II]-emission should be very small. This has been questioned by Petuchowski \& Bennett (1993) who accounted for about half of the [C II]-emission from PDRs, the other half from the WIM and only a very small fraction from the CNM. In contrast, Heiles (1994) found that the CNM ranks second after the WIM while PDR contributions should be small. Carral et al. (1994) state that up to $30 \%$ of the interstellar [C II] emission may stem from H II-regions. Kramer et al. (2005) used ISO/LWS observations of the [N II] $122 \mu \mathrm{m}$ line in the spiral arms of M 83 and M 51 to estimate that between $15 \%$ and $30 \%$ of the observed [C II] emission originates from H II-regions. Computations by Abel et al. (2005) indicate that the main [C II]emission arises from PDRs and a fraction of $10 \%$ up to $60 \%$ from H II-regions. Calculations by Kaufman et al. (2006) find similar [C II]-emission fractions, deviating by less than a factor of 2 .

We can test this result from the viewpoint of the excitation of $\mathrm{C}^{+}$which is a simple problem because the fine structure line arises basically from a two-level system. Using the excitation rates for collisions with $\mathrm{H}, \mathrm{H}_{2}, \mathrm{He}$, and electrons from Flower \& Launay (1977), Launay \& Roueff (1977), and Wilson \& Bell (2002) we can compute an upper limit to the emissivity of $\mathrm{C}^{+}$ as a function of density under the temperature and ionization conditions of the different phases of the ISM via

$\eta_{\mathrm{esc}}=\frac{1}{4 \pi} \frac{n_{\mathrm{C}^{+}} A_{\mathrm{ul}} E_{\mathrm{ul}} \beta}{1+\frac{g_{1}}{g_{\mathrm{u}}} \exp \left(\frac{E_{\mathrm{ul}}}{\mathrm{k}_{\mathrm{B}} \mathrm{T}}\right)\left(1+\frac{n_{\mathrm{c} r} \beta}{n}\right)}$

with $n$ the total number density of the collision partners (mainly hydrogen atoms or electrons), $A_{\mathrm{ul}}$ the Einstein coefficient for spontaneous emission, $g_{1, \mathrm{u}}$ the statistical weights for the lower
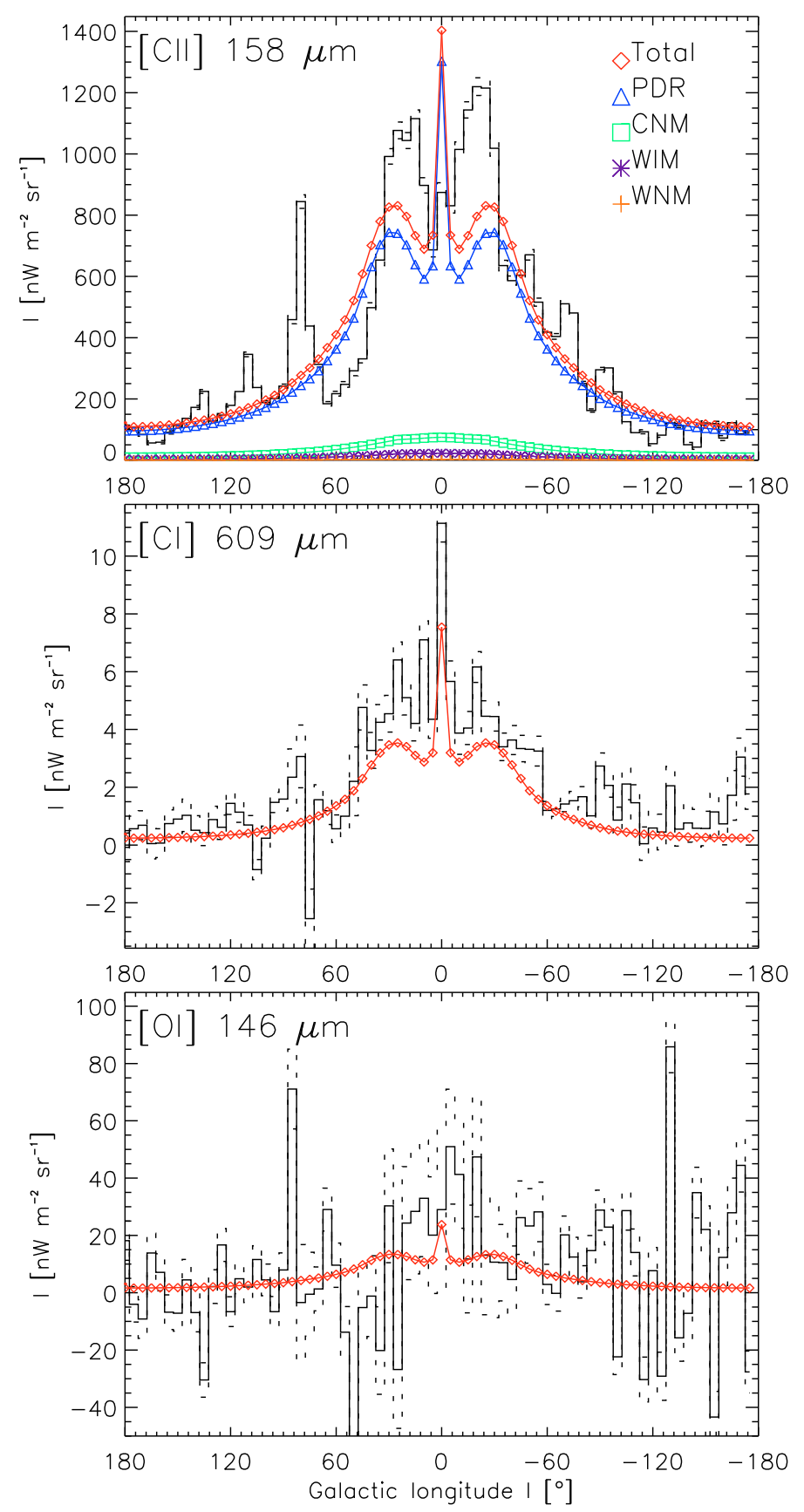

Fig. 9. The model results of the [C II] $158 \mu \mathrm{m}$, the [C I] $609 \mu \mathrm{m}$, and the [O I] $146 \mu \mathrm{m}$ intensity distributions with Galactic longitude overlaid on the observed distributions.

and upper state, $n_{\mathrm{C}^{+}}$the number density of $\mathrm{C}^{+}, E_{\mathrm{ul}}$ the energy spacing between the upper and lower state, $n_{\text {cr }}$ the critical density of the $[\mathrm{C}$ II $]{ }^{2} \mathrm{P}_{3 / 2}-{ }^{2} \mathrm{P}_{1 / 2}$ transition with respect to neutral particles or $n_{\mathrm{cr}, \mathrm{e}}$ for electrons, $k_{\mathrm{B}}$ the Boltzmann constant, and $T$ the temperature. The escape probability $\beta$ describes the probability of a photon of the considered transition to escape the cloud from its position within, assuming constant excitation conditions allover the cloud. To obtain an upper limit of the emission of the atomic gas components, we assume optically thin emission, i.e. $\beta=1$. The values for the different parameters are summarized in Table 5.

Figure 11 shows the [CII] emission from the different phases. All carbon is considered to be singly ionized, using an elemental abundance of $1.4 \times 10^{-4}$ (see Table 3 ). Upper limits are 

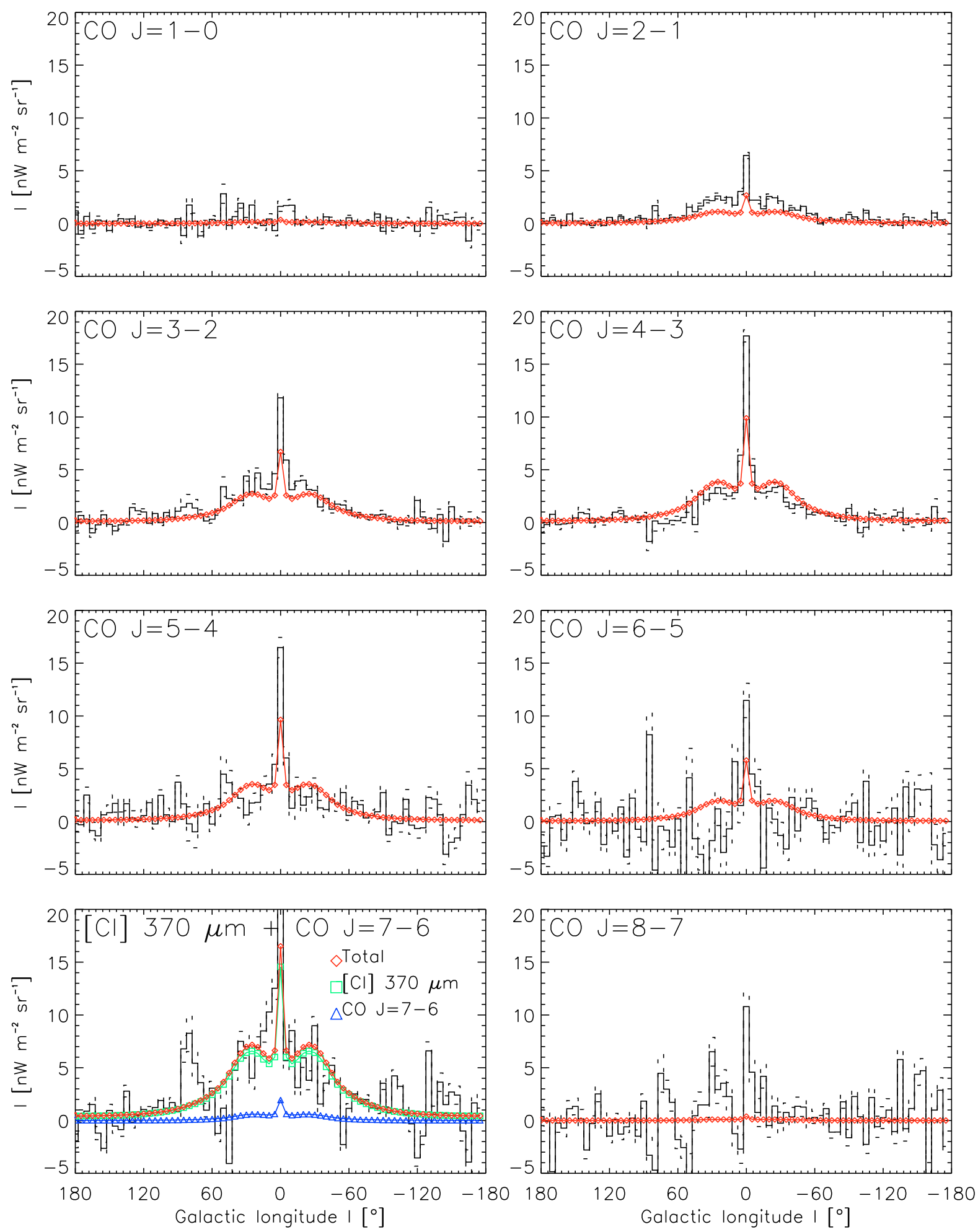

Fig. 10. The modeled intensities of the rotational transitions $J=1-0$ (upper left) to $J=8-7$ (lower right) of CO overlaid the observational distributions along the Galactic plane. Note that the combined CO $J=7-6$ and [C I] $370 \mu \mathrm{m}$ line intensities are shown in the lower left as they are not spectrally resolved by the COBE FIRAS instrument.

also guaranteed by assuming an ionization degree, i.e. an electron density relative to the total density of protons, of $10^{-3}$ in the CNM, $10^{-2}$ in the WNM, and 1 in the WIM (see Table 4 for the local Galactic parameter values of the different phases). We find that the "standard value" of $5 \times 10^{-26} \mathrm{erg} \mathrm{s}^{-1} \mathrm{H}_{\text {-atom }}^{-1}$ from Hollenbach \& Tielens (1999) is in agreement with the density range of the CNM given in Wolfire et al. (2003). At low densities an efficient excitation of $\mathrm{C}^{+}$is only possible if a large fraction of the collision partners is charged, i.e. in the ionized medium.

The results of Petuchowski \& Bennett (1993) and Abel (2006) who found, that the [C II] and [N II] emission are strongly correlated, indicate that main contributions ( $60 \%$ maximum) are expected from low density H II-regions. Using this result, we 


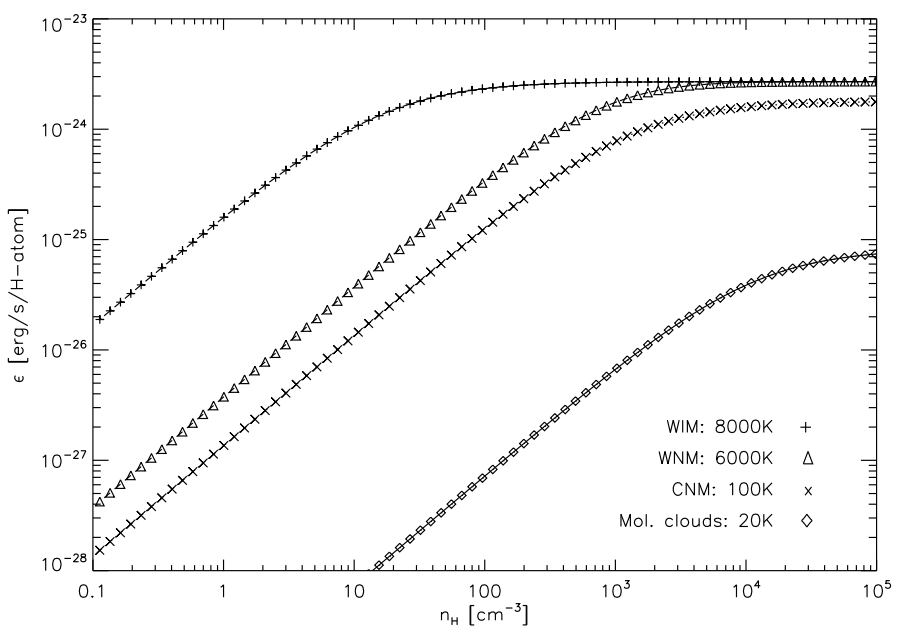

Fig. 11. Optically thin emissivity of [C II] under the excitation conditions of the different ISM components as a function of the gas density.

Table 5. The parameter values used for the escape probability calculation of the $[\mathrm{CII}]{ }^{2} \mathrm{P}_{3 / 2}-{ }^{2} \mathrm{P}_{1 / 2}$ emissivity from the CNM and WNM. Powers of ten are given in parentheses.

\begin{tabular}{lr}
\hline \hline Quantity & Value \\
\hline$\beta$ & 1 \\
$g_{\mathrm{u}}$ & $4 / 3$ \\
$g_{\mathrm{l}}$ & $2 / 3$ \\
$A_{\mathrm{ul}}\left[\mathrm{s}^{-1}\right]$ & $2.4(-6)$ \\
$n_{\mathrm{cr}}\left[\mathrm{cm}^{-3}\right]$ & $4(3)$ \\
$n_{\mathrm{cr}, \mathrm{e}}\left[\mathrm{cm}^{-3}\right]$ & $4(1)$ \\
\hline
\end{tabular}

rerun the $\chi^{2}$ fit of the lines but increase the computed [C II]intensity by a global factor 2.5 before fitting the model results to the observations. This fit provides a lower limit of the FUV-flux of $\chi=10^{1.2} \chi_{\mathrm{D}}$, which is slightly below the estimated range from Section 2.4 with a lower limit of $\chi=10^{1.3} \chi_{\mathrm{D}}$. The best fitting ensemble averaged clump density is derived to $n_{\mathrm{ens}}=10^{3.9} \mathrm{~cm}^{-3}$ in this case. Additionally we check the fit results completely ignoring the $[\mathrm{C} \mathrm{II}]$ intensity distribution. In this case we obtain parameter values of $n_{\mathrm{ens}}=10^{3.8} \mathrm{~cm}^{-3}$ and $\chi=10^{1.0} \chi_{\mathrm{D}}$. The $\chi^{2}$ distribution with a minimum of 5.40 for this case is also shown in Fig. 8. The resulting low FUV-flux is due to the low signal to noise ratio in the $[\mathrm{OI}]$ and upper $\mathrm{CO}$ transitions, so that this result is biased by the low- $J \mathrm{CO}$ and $[\mathrm{CI}$ ] emission and lies below our estimate for the average FUV-flux seen by the Galactic molecular gas. The confinement of the $\chi^{2}$ distribution ignoring the $[\mathrm{C} \mathrm{II}]$ intensity distribution is weak in either direction. In particular at $n_{\mathrm{ens}}=10^{3.8} \mathrm{~cm}^{-3}$ and $\chi=10^{1.8} \chi_{\mathrm{D}}$ we find a $\chi^{2}$ value of 5.75 - only about $7 \%$ more than the minimum value, indicating that the $[\mathrm{C} \mathrm{II}]$ emission fixes the resulting FUV-flux value of the model. The computed PDR [C II] intensity distributions for these cases are shown in Fig. 12.

There are smaller, comparable contributions from the diffuse WIM, which has an average density well below $1 \mathrm{~cm}^{-3}$ (McKee \& Ostriker 1977; Reynolds 1991; Cox 2005; Hill et al. 2007), and from the CNM , and a vanishing contribution of the WNM. However, the much larger scale height of the diffuse WIM leads to a reduced contribution in comparison to the CNM, in agreement with our result for the intensity distribution along the Galactic plane shown in Fig. 9.

We conclude that our model can reproduce the Galactic [C II] emission with the main contribution from clumpy PDRs, but

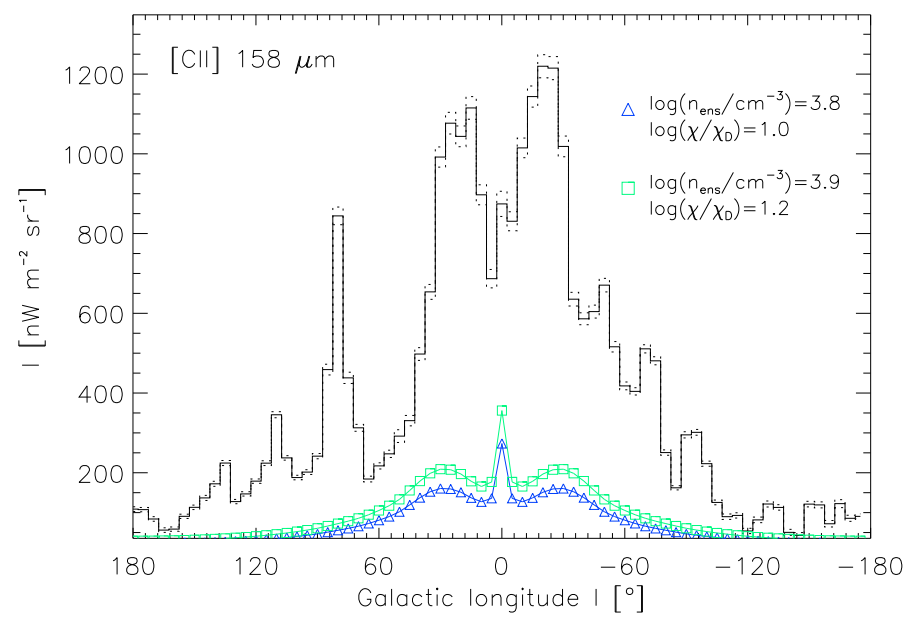

Fig. 12. PDR $[\mathrm{CII}]$ intensity corresponding to the best fit parameter values ignoring the $[\mathrm{CII}]$ emission and under the assumption that the contribution of the PDR-correlated $\mathrm{H}$ II regions to the total [C II] emission is $60 \%$.

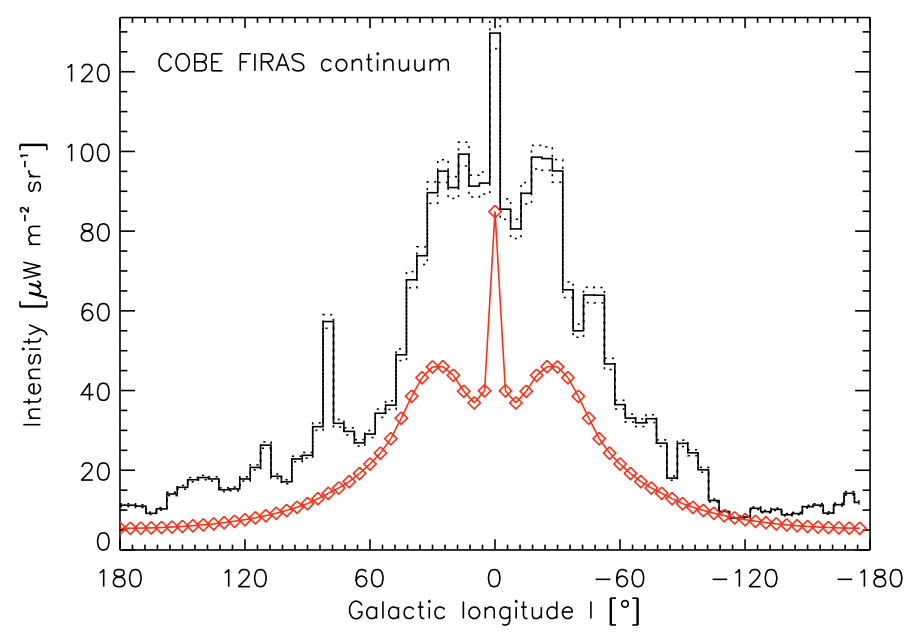

Fig. 13. The modeled FIR intensity distribution along the Galactic plane in the COBE FIRAS wavelength band overlaid on the observed distribution as published by Fixsen et al. (1999).

we cannot exclude a major contribution from low-density H II regions.

\subsubsection{The FIR continuum emission}

Only $51 \%$ of the Galactic FIR emission in the COBE FIRAS spectral range is reproduced by a separate dust radiative transfer calculation for the KOSMA- $\tau$ PDR-model clumps (R. Szczerba, priv. comm.) as shown in Fig. 13. This is expected, as the dust is also heated by less energetic radiation than the FUV from the older stellar population. Mochizuki \& Nakagawa (2000) argue that about half of the total stellar flux lies in the FUV spectral range in agreement with our result.

\section{Summary and conclusions}

The bulk of the FIR and submillimeter line emission in [C II] 158 $\mu \mathrm{m},[\mathrm{CI}] 609 \mu \mathrm{m}$ and $370 \mu \mathrm{m}$, [O I] $146 \mu \mathrm{m}$, and CO $1-0$ to 8-7 of the Milky Way as observed by COBE FIRAS can be reproduced by a clumpy PDR-model, which takes the fractal structure of the dense ISM as a clump ensemble with a power law clump 
mass and size distribution into account. This is a remarkable result, as the model contains essentially no free parameters; all parameters being constrained by independent knowledge about the structure, kinematics of, and star formation rate in the disk of the Milky Way.

The result suggests that the bulk of the Galactic FIR line emission stems from fractally structured PDRs, illuminated by the FUV radiation field emitted by the Galactic population of massive stars in adjacent star forming regions.

The UV-penetrated, clump-cloud PDR scenario applied here to the Milky Way line emission as observed by COBE is a versatile approach, which can be applied in the future to model the emission from individual Galactic star forming regions as well as the line maps of spatially resolved nearby galaxies.

Future studies and observations should address the validity of particular assumptions, like the limits of the clump mass distribution. Observations with high spatial and/or spectral resolution, that will be possible with upcoming missions as SOFIA, Herschel, and ALMA, will give further insights in the nature of the ISM.

Acknowledgements. We are grateful to D. J. Fixsen for providing us the results of Fixsen et al. (1999) in electronic form. We thank the anonymous referee for her/his helpful comments. This work is supported by the Deutsche Forschungsgemeinschaft (DFG) via Grant SFB 494.

\section{References}

Abel, N. P. 2006, MNRAS, 368, 1949

Abel, N. P., Ferland, G. J., Shaw, G., \& van Hoof, P. A. M. 2005, ApJS, 161, 65 Anders, E., \& Grevesse, N. 1989, Geochim. Cosmochim. Acta, 53, 197 Bennett, C. L., Fixsen, D. J., Hinshaw, G., et al. 1994, ApJ, 434, 587

Blaauw, A. 1985, in The Milky Way Galaxy, ed. H. van Woerden, R. J. Allen, \& W. B. Burton, IAU Symposium, 106, 335

Boreiko, R. T., \& Betz, A. L. 1991, ApJ, 369, 382

Brand, J., \& Wouterloot, J. G. A. 1995, A\&A, 303, 851

Bronfman, L., Casassus, S., May, J., \& Nyman, L.-A. 2000, A\&A, 358, 521

Carral, P., Hollenbach, D. J., Lord, S. D., et al. 1994, ApJ, 423, 223

Clemens, D. P. 1985, ApJ, 295, 422

Contursi, A., Kaufman, M. J., Helou, G., et al. 2002, AJ, 124, 751

Cox, D. P. 2005, ARA\&A, 43, 337

Dahmen, G., Hüttemeister, S., Wilson, T. L., \& Mauersberger, R. 1998, A\&A, 331,959

Dame, T. M., Hartmann, D., \& Thaddeus, P. 2001, ApJ, 547, 792

Draine, B. T. 1978, ApJS, 36, 595

Falgarone, E., Panis, J.-F., Heithausen, A., et al. 1998, A\&A, 331, 669

Fixsen, D. J., Bennett, C. L., \& Mather, J. C. 1999, ApJ, 526, 207

Flower, D. R., \& Launay, J. M. 1977, J. Phys. B Atom. Mol. Phys., 10, 3673

Gierens, K. M., Stutzki, J., \& Winnewisser, G. 1992, A\&A, 259, 271

Gorti, U., \& Hollenbach, D. 2002, ApJ, 573, 215

Graf, U. U., Genzel, R., Harris, A. I., et al. 1990, ApJ, 358, L49

Gry, C., Lequeux, J., \& Boulanger, F. 1992, A\&A, 266, 457

Güsten, R., \& Philipp, S. D. 2004, in The Dense Interstellar Medium in Galaxies, ed. S. Pfalzner, C. Kramer, C. Straubmeier, \& A. Heithausen, 253

Habing, H. J. 1968, Bull. Astron. Inst. Netherlands, 19, 421

Heiles, C. 1994, ApJ, 436, 720

Heithausen, A., Bensch, F., Stutzki, J., Falgarone, E., \& Panis, J. F. 1998, A\&A, 331, L65
Hill, A. S., Reynolds, R. J., Benjamin, R. A., \& Haffner, L. M. 2007, in SINS Small Ionized and Neutral Structures in the Diffuse Interstellar Medium, ed. M. Haverkorn, \& W. M. Goss, ASP Conf. Ser., 365, 250

Hollenbach, D. J., \& Tielens, A. G. G. M. 1999, Rev. Mod. Phys., 71, 173

Howe, J. E., Jaffe, D. T., Genzel, R., \& Stacey, G. J. 1991, ApJ, 373, 158

Jaffe, D. T., Harris, A. I., \& Genzel, R. 1987, ApJ, 316, 231

Kaufman, M. J., Wolfire, M. G., Hollenbach, D. J., \& Luhman, M. L. 1999, ApJ, 527,795

Kaufman, M. J., Wolfire, M. G., \& Hollenbach, D. J. 2006, ApJ, 644, 283

Köster, B., Störzer, H., Stutzki, J., \& Sternberg, A. 1994, A\&A, 284, 545

Kramer, C., Stutzki, J., Rohrig, R., \& Corneliussen, U. 1998, A\&A, 329, 249

Kramer, C., Jakob, H., Mookerjea, B., et al. 2004, A\&A, 424, 887

Kramer, C., Mookerjea, B., Bayet, E., et al. 2005, A\&A, 441, 961

Kramer, C., Cubick, M., Röllig, M., et al. 2008, A\&A, 477, 547

Launay, J. M., \& Roueff, E. 1977, A\&A, 56, 289

Madden, S., Geis, N., Genzel, R., et al. 1997, in ESA Special Publication, The Far Infrared and Submillimetre Universe., ed. A. Wilson, 401, 111

Malhotra, S., Kaufman, M. J., Hollenbach, D., et al. 2001, ApJ, 561, 766

McKee, C. F., \& Ostriker, J. P. 1977, ApJ, 218, 148

McKee, C. F., \& Williams, J. P. 1997, ApJ, 476, 144

Melnick, G., Gull, G. E., \& Harwit, M. 1979, ApJ, 227, L29

Menten, K. M., Reid, M. J., Forbrich, J., \& Brunthaler, A. 2007, A\&A, 474, 515

Misiriotis, A., Xilouris, E. M., Papamastorakis, J., Boumis, P., \& Goudis, C. D. 2006, A\&A, 459, 113

Mizutani, K., Maihara, T., Matsuhara, H., et al. 1994, ApJS, 91, 613

Mochizuki, K., \& Nakagawa, T. 2000, ApJ, 535, 118

Nikola, T., Genzel, R., Herrmann, F., et al. 1998, ApJ, 504, 749

Petuchowski, S. J., \& Bennett, C. L. 1993, ApJ, 405, 591

Pottasch, S. R., Wesselius, P. R., \& van Duinen, R. J. 1979, A\&A, 74, L15

Reynolds, R. J. 1990, in The Galactic and Extragalactic Background Radiation, ed. S. Bowyer, \& C. Leinert, IAU Symp., 139, 157

Reynolds, R. J. 1991, ApJ, 372, L17

Rohlfs, K., \& Kreitschmann, J. 1987, A\&A, 178, 95

Röllig, M., Ossenkopf, V., Jeyakumar, S., Stutzki, J., \& Sternberg, A. 2006, A\&A, 451, 917

Röllig, M., Abel, N. P., Bell, T., et al. 2007, A\&A, 467, 187

Russell, R. W., Melnick, G., Gull, G. E., \& Harwit, M. 1980, ApJ, 240, L99

Russell, R. W., Melnick, G., Smyers, S. D., et al. 1981, ApJ, 250, L35

Schilke, P., Carlstrom, J. E., Keene, J., \& Phillips, T. G. 1993, ApJ, 417, L67

Simon, R., Jackson, J. M., Clemens, D. P., Bania, T. M., \& Heyer, M. H. 2001, ApJ, 551, 747

Stacey, G. J., Smyers, S. D., Kurtz, N. T., \& Harwit, M. 1983, ApJ, 265, L7

Stark, A. A., \& Blitz, L. 1978, ApJ, 225, L15

Sternberg, A., \& Dalgarno, A. 1989, ApJ, 338, 197

Storey, J. W. V., Watson, D. M., Townes, C. H., Haller, E. E., \& Hansen, W. L. 1981, ApJ, 247, 136

Störzer, H., Stutzki, J., \& Sternberg, A. 1996, A\&A, 310, 592

Stutzki, J., \& Güsten, R. 1990, ApJ, 356, 513

Stutzki, J., Stacey, G. J., Genzel, R., et al. 1988, ApJ, 332, 379

Stutzki, J., Bensch, F., Heithausen, A., Ossenkopf, V., \& Zielinsky, M. 1998, A\&A, 336, 697

Tielens, A. G. G. M., \& Hollenbach, D. 1985, ApJ, 291, 722

Unger, S. J., Clegg, P. E., Stacey, G. J., et al. 2000, A\&A, 355, 885

Watson, D. M., Genzel, R., Townes, C. H., \& Storey, J. W. V. 1985, ApJ, 298, 316

Williams, J. P., \& Blitz, L. 1993, ApJ, 405, L75

Williams, J. P., \& McKee, C. F. 1997, ApJ, 476, 166

Williams, J. P., de Geus, E. J., \& Blitz, L. 1994, ApJ, 428, 693

Wilson, N. J., \& Bell, K. L. 2002, MNRAS, 337, 1027

Wolfire, M. G., Hollenbach, D., \& Tielens, A. G. G. M. 1989, ApJ, 344, 770

Wolfire, M. G., McKee, C. F., Hollenbach, D., \& Tielens, A. G. G. M. 2003, ApJ, 587,278

Wright, E. L., Mather, J. C., Bennett, C. L., et al. 1991, ApJ, 381, 200 